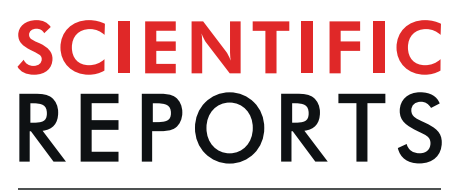

natureresearch

\title{
PtomtAPX, a mitochondrial ascorbate peroxidase, plays an important role in maintaining the redox balance of Populus tomentosa Carr
}

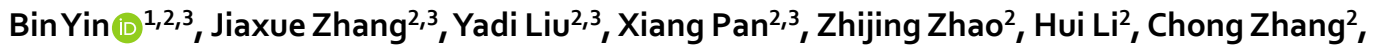
Conghui $\mathrm{Li}^{2}$, Xihua $\mathrm{Du}^{2}$, Yinjun $\mathrm{Li}^{2}$, Di Liu ${ }^{2 *} \& \mathrm{Hai} \mathrm{Lu} \mathbb{B}^{1,2^{*}}$

Plant mitochondria are important energy-producing structure and ROS are generated as byproducts. APX is one enzyme of the AsA-GSH cycle to reduces $\mathrm{H}_{2} \mathrm{O}_{2}$ to water. We identified both PtomtAPX and PtosAPX are located in mitochondria of Populus tomentosa Carr. PtomtAPX is specifically targeted to mitochondria, while PtosAPX is dual targeted to both chloroplast and mitochondria. The expression of PtomtAPX in mitochondria was 60-fold that of PtosAPX by ELISA and qPCR analysis. Under high light stress, the expression levels of PtosAPX increased, while that of PtomtAPX only slightly changed. Compared to the WT, the antisense transgenic PtomtAPX cell lines showed slowed growth, smaller cells impaired mitochondria in MS medium under normal growth. RNA-seq results showed 3121 genes significantly altered expression in the antisense cells, and most of them are important for mitochondrial function, particularly in oxidative phosphorylation. Our findings demonstrates a mitochondrial location for one APX isoform, and provide valuable insight into the mechanism which ROS balance is modulated by AsA-GSH cycle in mitochondria.
\end{abstract}

Reactive oxygen species (ROS) are generated as byproducts of normal cell metabolism in several organelles and their production is enhanced under stress conditions ${ }^{1}$. APX is one enzyme of the ascorbate-glutathione cycle (AsA-GSH cycle) in plants ${ }^{2,3}$. APX in Arabidposis are localized to the cytosol (cAPX, AT1G07890, AT3G09640, AT4G32320), chloroplast (thylakoid-bound APX [tAPX, AT1G77490] and stromal APX [sAPX, AT4G08390]), microbody (including the peroxisome and glyoxisome) (mAPX, AT4G35000, AT4G35970) by organelle-specific targeting peptides and transmembrane domains ${ }^{4-7}$ and to remove $\mathrm{H}_{2} \mathrm{O}_{2}$ in the organelles themselves ${ }^{2}$. In Arabidopsis, apx1(AT1G07890) knockout plants showed increased $\mathrm{H}_{2} \mathrm{O}_{2}$ levels, higher sensitivity to oxidative stress, and suppressed growth and development ${ }^{8-10}$. Arabidopsis apx3(AT4G35000) knockout mutants did not show suppressed growth under normal or stress conditions ${ }^{11}$. Arabidopsis double tapx(AT1G77490)/apx1 (AT1G07890) mutants showed late flowering, low protein oxidation during light stress and enhanced accumulation of anthocyanins ${ }^{12}$. The levels of ROS were increased and the germination was reduced in seeds of Arabidopsis APX6 knockout mutants ${ }^{13}$. Rice plants double silenced for cytosolic APXs showed normal growth and development and were able to survive under stress conditions ${ }^{14,15}$. Loss of function in OsAPX2(Os07 g0694700) showed semi-dwarf seedlings, yellow-green leaves and seed sterility ${ }^{16}$. Rice peroxisomal ascorbate peroxidase(OsAPX4; Os08g43560) knockdown showed early leaf senescence ${ }^{17}$. These results indicate that the APXs isoenzymes are indispensable for plant growth and development.

Jimenez et al. (1998) reported APX activity in the external side of the outer mitochondria membrane from $\mathrm{pea}^{18}$.Three different APX isoforms was showed in mitochondria of tomato (Lycopersicon esculentum) using native gel electrophoresis ${ }^{19}$. De Leonardis et al. ${ }^{20}$ reported high APX activity which maybe localized inside

${ }^{1}$ Beijing Advanced Innovation Center for Tree Breeding by Molecular Design, Beijing Forestry University, Beijing, 100083, People's Republic of China. ${ }^{2}$ College of Biological Sciences and Biotechnology, Beijing Forestry University, Beijing, 100083, People's Republic of China. ${ }^{3}$ These authors contributed equally: Bin Yin, Jiaxue Zhang, Yadi Liu and Xiang Pan. *email: liudi@bjfu.edu.cn; luhai1974@bjfu.edu.cn 
mitochondria using sonication-mediated disruption of potato mitochondria ${ }^{20}$. And the presence of more than one APX in mitochondria of both leaves and young green inflorescences of Chenopodium album was detected by native gel electrophoresis ${ }^{21}$. However, until now, no gene, cDNA, or protein sequence for the plant specifically mitochondrial isoform (mitAPX) has been described. Arabidopsis single or double chlAPX (sAPX and tAPX) mutants showed normal phenotype under normal growth conditions or under high light intensity stress growth conditions $^{22-24}$. In addition, sAPX knockdown rice plants exhibit a normal phenotype and show normal biochemical and physiological performance under normal growth conditions ${ }^{25}$. These results suggest that sAPX is not important for $\mathrm{H}_{2} \mathrm{O}_{2}$ scavenging in chloroplasts and/or mitochondria of $A$. thaliana or rice.

In this study, we investigated there is a mitochondria-specific APX of $P$. tomentosa using green fluorescent protein (GFP) fusion experiments and immunoelectron microscopy. And PtosAPX is dual targeted to both chloroplast and mitochondria. The expression levels of PtomtAPX and PtosAPX were modulated by $\mathrm{H}_{2} \mathrm{O}_{2}, \mathrm{NaCl}$, heat, drought, and cold. Compared to the WT, the antisense transgenic PtomtAPX cell lines showed slowed growth, smaller cells impaired mitochondria in MS medium under normal growth. The results indicated that PtomtAPX is specifically targeted to mitochondria and plays an important role in maintaining the redox balance in Populus tomentosa Carr.

\section{Results}

PtomtAPX is specifically targeted to mitochondria, and PtosAPX is targeted to both chloroplasts and mitochondria. The poplar database (the JGI Populus trichocarpav.1.1 genome browser; http:// genome.jgi-psf.org/Poptr1_1/Poptr1_1.home.html; Tuskan et al. (2006)) was searched and 10 genes are predicted as APX proteins, and 2 genes(Protein Id: 209946, 798682) was speculated as putative mitochondrial/chloroplasts APX which has a mitochondria/chloroplast localization signal peptide. These homologous APX in P. tomentosa were cloned using primers specific for the Populus trichocarpa APX gene (Protein ID: 209946, 798682), respectivity. A 1,080 bp open reading frame (ORF) (homologous APX in Populus trichocarpa, Protein ID: 798682. Supplemental Fig. 1A) was isolated, sequenced, and named PtomtAPX, and a 1,086 bp ORF (homologous APX in Populus trichocarpa, Protein ID: 209946. Supplemental Fig. 1B) was isolated, sequenced, and named PtosAPX.

Sequence alignment revealed the presence of an N-terminal mitochondria/chloroplast-targeting peptide and two signatures of chloroplastic isoforms (KNIEEWP and ETKYTKDGPGAPGGQS) in PtosAPX and PtomtAPX, respectively (Fig. 1A and Supplemental Fig. 1). Phylogenetic analyses of APXs indicated that PtomtAPX and PtosAPX were chloroplastic and/or mitochondrial isoforms (Supplemental Fig. 2). Positively charged amino acid residues and amphipathic $\alpha$-helix within the $19 \mathrm{~N}$-terminal portion of the targeting peptide are important for the importation of proteins into mitochondria but not chloroplasts ${ }^{26}$. PtomtAPX had four positively charged residues and two amphiphilic $\alpha$-helices in the targeting peptide, while PtosAPX had no positively charged residue and no amphiphilic $\alpha$-helix (Fig. 1A). These results suggest that PtomtAPX is a mitochondrial isoform.

The subcellular localization of PtosAPX and PtomtAPX in P. tomentosa was determined by fusing their full-length coding sequences upstream of GFP under the control of the $35 \mathrm{~S}$ promoter (Fig. 1). The GFP signal of PtosAPX-GFP was detected not only in mitochondria co-stained with MitoTracker Red CMXRos (hereafter, CMXRos) but also in chloroplasts (red autofluorescence replaced by blue pseudocolor) in leaf epidermal cells. The GFP fluorescence of PtomtAPX-GFP was detected in mitochondria in leaf epidermal cells and root tips but not in chloroplasts.

Immunoelectron microscopy was performed to confirm the localization of PtosAPX and PtomtAPX. Immunogold labeling using rabbit anti-PtosAPX and anti-PtomtAPX antibodies showed that PtosAPX was localized to mitochondria and chloroplasts and PtomtAPX was localized to mitochondria (Fig. 1M-P). Taken together, these results suggest that PtomtAPX is localized to mitochondria in P. tomentosa, while PtosAPX is localized to both mitochondria and chloroplasts and shows greater similarity to a chloroplastic isoform.

PtomtAPX and PtosAPX expression in mitochondria. The presence of PtomtAPX and PtosAPX in mitochondria was investigated by enzyme-linked immunosorbent assay (ELISA). PtosAPX was detected in both mitochondria and chloroplasts. The PtosAPX level in chloroplasts was 78-fold that in mitochondria (Fig. 2A) and the PtomtAPX level in mitochondria was 60-fold that of PtosAPX (Fig. 2B). Therefore, PtomtAPX is the primary APX in mitochondria, while PtosAPX is likely to be a chloroplastic isoform.

The expression levels of PtomtAPX and PtosAPX in the presence of $\mathrm{H}_{2} \mathrm{O}_{2}, \mathrm{NaCl}$, heat, drought, cold, or high-intensity light were determined by qRT-PCR with normalization to the actin gene (Potri.001G309500). Under high-light stress, the expression levels of PtosAPX increased and decreased, respectively, while that of PtomtAPX only slightly changed (Fig. 2C,D). The expression levels of PtomtAPX and PtosAPX were modulated by $\mathrm{H}_{2} \mathrm{O}_{2}, \mathrm{NaCl}$, heat, drought, and cold.

Enzymatic characteristics of PtomtAPX and PtosAPX. The expression constructs $p E T 30 a-P$ tomtAPX and $p E T 30 a-P t o s A P X$, which harbored the full-length cDNA minus the signal peptide, were expressed in Escherichia coli, resulting in production of recombinant PtomtAPX and PtosAPX, respectively. Sodium dodecyl sulfate (SDS)-PAGE showed that the molecular weight of purified recombinant PtomtAPX and PtosAPX were both $39 \mathrm{kDa}$. The reaction of PtomtAPX or PtosAPX with AsA and $\mathrm{H}_{2} \mathrm{O}_{2}$ followed Michaelis-Menten kinetics. At a fixed AsA concentration, the $K_{\mathrm{m}}$ and $V_{\max }$ values of PtomtAPX were $0.03 \pm 0.00 \mathrm{mM}$ and $1.54 \pm 0.00 \mathrm{mM} \mathrm{min}^{-1} \mathrm{mg}^{-1}$ for $\mathrm{H}_{2} \mathrm{O}_{2}$, respectively, while the $K_{\mathrm{m}}$ and $V_{\max }$ values of PtosAPX were $0.04 \pm 0.01 \mathrm{mM}$ and $1.64 \pm 0.11 \mathrm{mmol} \cdot \mathrm{min}^{-1} \cdot \mathrm{mg}^{-1}$ for $\mathrm{H}_{2} \mathrm{O}_{2}$, respectively. At a fixed $\mathrm{H}_{2} \mathrm{O}_{2}$ concentration, the $K_{\mathrm{m}}$ and $V_{\max }$ values of PtomtAPX were $6.04 \pm 0.06 \mathrm{mM}$ and $3.00 \pm 0.14 \mathrm{mM} \mathrm{min}^{-1} \mathrm{mg}^{-1}$ for AsA, respectively, while the $K_{\mathrm{m}}$ and $V_{\max }$ values of PtosAPX were $4.30 \pm 1.81 \mathrm{mM}$ and $0.85 \pm 0.25 \mathrm{mmol} \cdot \mathrm{min}^{-1} \cdot \mathrm{mg}^{-1}$ for AsA, respectively (Table 1). PtomtAPX and PtosAPX exhibited similar turnover rates $\left(k_{\text {cat }}\right)$, efficiencies $\left(k_{\text {cat }} / K_{\mathrm{m}}\right)$ and affinities $\left(K_{\mathrm{m}}\right)$ for $\mathrm{H}_{2} \mathrm{O}_{2}$ and AsA. Therefore, PtomtAPX and PtosAPX are APX enzymes with similar activities. 


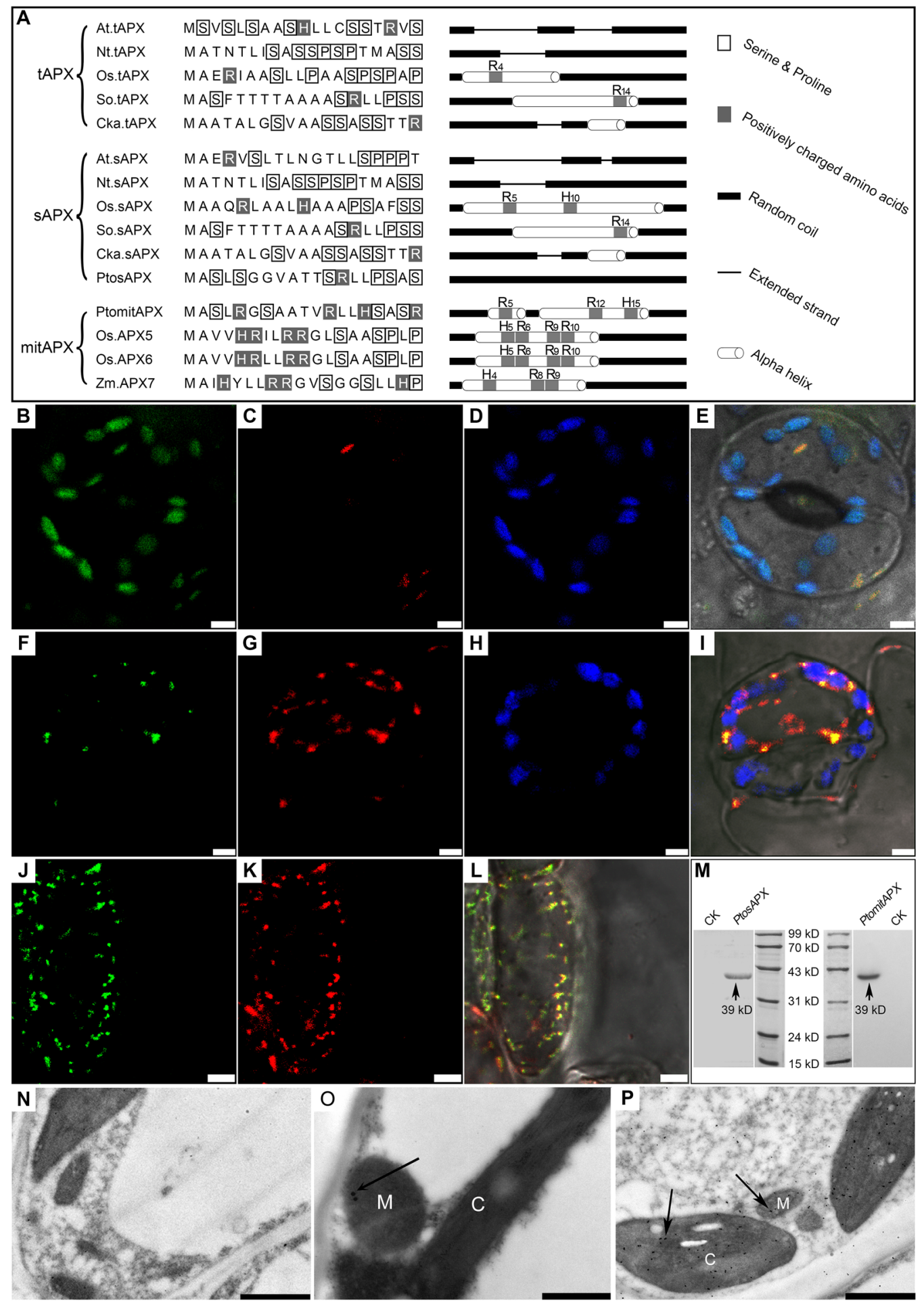

Figure 1. Amino acid sequence and subcellular distribution of PtomtAPX and PtosAPX. (A) Targeting peptide alignment and secondary structure analyses. (B-E) Expression of the PtosAPX-GFP fusion protein in leaf epidermal cells of transgenic tobacco. (B) Fluorescence of PtosAPX-GFP fusion protein. (C) Mitochondria stained with MitoTracker Red. (D) Chloroplast autofluorescence. (E) Merged image. (F-L) Expression of the PtomtAPX-GFP fusion protein in leaf epidermal and root-tip cells of transgenic tobacco. (F,J) Fluorescence of PtomtAPX-GFP fusion protein. (G) and (K) Mitochondria stained with MitoTracker Red. (H) Chloroplast autofluorescence. (I,L) Merged images. Bars, $5 \mu \mathrm{m}$. (M) Western blotting analyses of total protein extracts from leaf cells of $P$. tomentosa using anti-PtomtAPX and anti-PtosAPX antibodies. (N) Negative control. (O) Immunoelectron microscopy of PtomtAPX. (P) Immunoelectron microscopy of Ptos APX. $1 \mu \mathrm{m}(\mathbf{N}-\mathbf{P})$. M, mitochondrion, $\mathrm{C}$, chloroplast. 


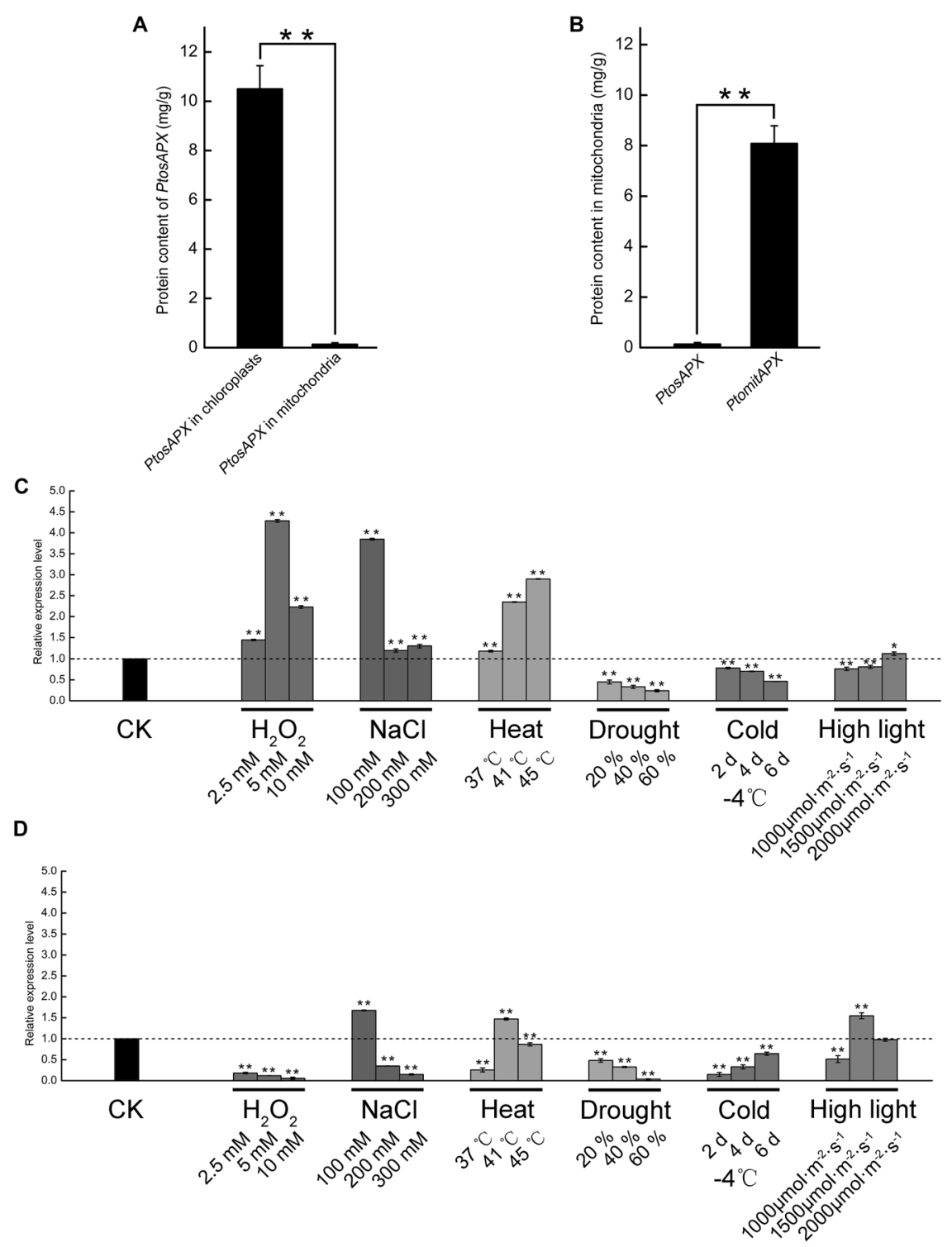

Figure 2. Expression profile of PtosAPX and PtomtAPX. (A) PtosAPX level in mitochondria and chloroplasts according to ELISA. (B) PtosAPX and PtomtAPX levels in mitochondria according to ELISA. (C-E) Expression of PtomtAPX and PtosAPX under abiotic stress according to qRT-PCR. (C) PtomtAPX. (D) PtosAPX.

**Significantly different at $\mathrm{P}<0.01$ and *Significantly different at $\mathrm{P}<0.05$. Bars, standard deviations.

\begin{tabular}{|l|l|l|l|l|l|}
\hline APX isoforms & Substrate & $\boldsymbol{K}_{\mathbf{m}}(\mathbf{m M})$ & $\boldsymbol{V}_{\max }\left(\mathbf{m M} \mathbf{m i n}^{-1}\right)$ & $\boldsymbol{k}_{\text {cat }}\left(\mathbf{m i n}^{-1}\right)$ & $\boldsymbol{k}_{\text {cat }} / \boldsymbol{K}_{\mathbf{m}}\left(\mathbf{m M}^{-1} \mathbf{m i n}^{-1}\right)$ \\
\hline \multirow{2}{*}{ PtomtAPX } & ASA & $6.04 \pm 0.06$ & $3.00 \pm 0.14$ & 928405.63 & 153709.54 \\
\cline { 2 - 6 } & $\mathrm{H}_{2} \mathrm{O}_{2}$ & $0.03 \pm 0.00$ & $1.54 \pm 0.00$ & 475149.03 & 15838330.11 \\
\hline \multirow{2}{*}{ PtosAPX } & $\mathrm{ASA}$ & $4.30 \pm 1.81$ & $0.85 \pm 0.25$ & 897148.78 & 208505.07 \\
\cline { 2 - 6 } & $\mathrm{H}_{2} \mathrm{O}_{2}$ & $0.04 \pm 0.01$ & $1.64 \pm 0.11$ & 864716.73 & 20312959.95 \\
\hline
\end{tabular}

Table 1. Enzymatic properties of recombinant PtomtAPX and PtosAPX. Values are means \pm SDs of three replicates.

Decreased PtomtAPX content leads to mitochondrial dysfunction and PCD. We used a pBI121-based construct to generate 45 antisense-PtomtAPX transgenic $P$. tomentosa suspension cell lines. The PtomtAPX transcript levels were 5-95\% lower in 10 randomly selected PtomtAPX transgenic P. tomentosa cell lines compared to the WT. Three representative PtomtAPX transgenic cell lines with PtomtAPX transcript levels $61.63 \%, 34.29 \%$, and 5.24\% lower than the WT were named anti-1, anti-2, and anti-3, respectively (Fig. 3A). The PtomtAPX protein level was $67.18 \%, 41.55 \%$, and $7.68 \%$ lower in anti-1, anti-2, and anti-3, respectively, than in the WT (Fig. 3B). Compared to the WT, the three transgenic PtomtAPX cell lines showed slowed growth and 
A

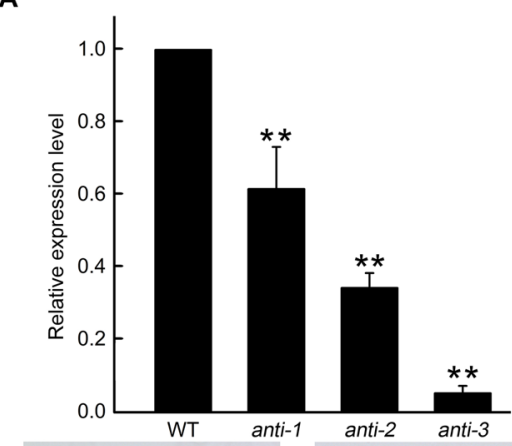

C

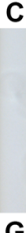

G

K
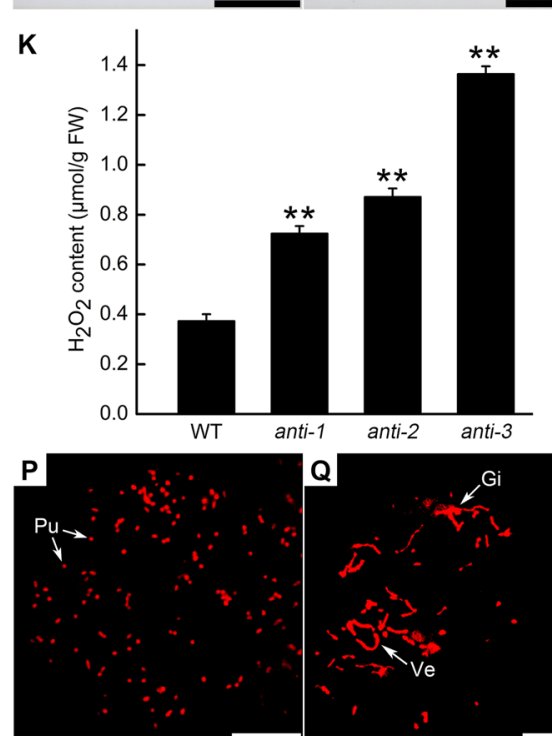

$\mathbf{R}$

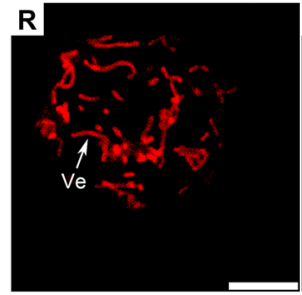

$\mathbf{Q}$

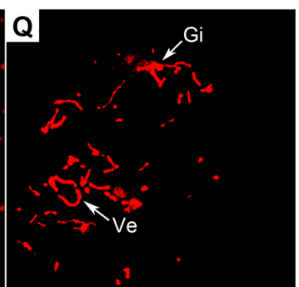

s
D

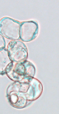

B

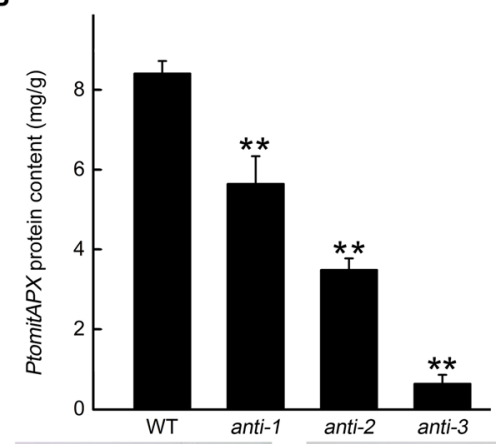

E

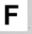

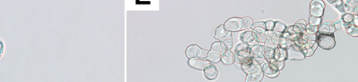

$F$
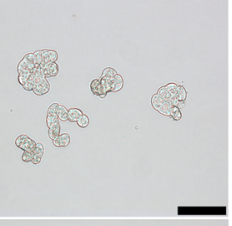

I
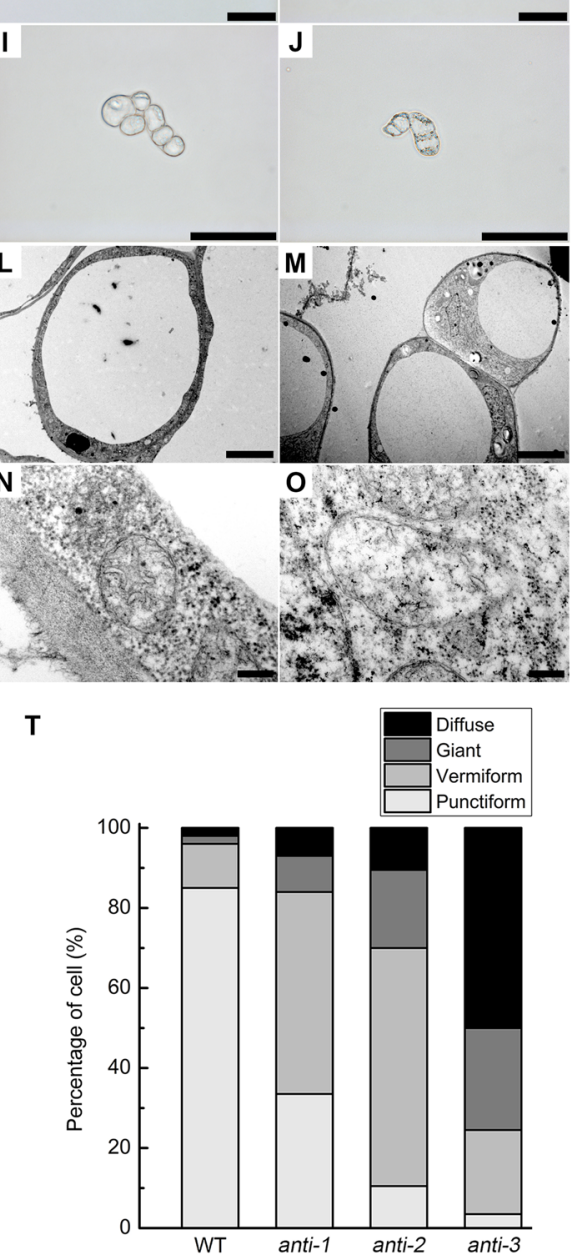

Figure 3. PtomtAPX expression, cell morphology and mitochondrial structure of PtomtAPX-antisense suspension cells. (A) qRT-PCR. (B) ELISA. **Significantly different at P $<0.01$; bars represent standard deviations. (C-J) Cell morphology. (C,G) Morphology of WT; (D,H) anti-1; (E,I) anti-2, (F,J) anti-3. Bars, $100 \mu \mathrm{m}$. (K) Mitochondrial $\mathrm{H}_{2} \mathrm{O}_{2}$ content. (L-O) TEM of cell morphology and mitochondrial morphology. (L,N) WT; (M,O) PtomtAPXantisense. Bars, $5 \mu \mathrm{m}$ in (L,M) and $200 \mathrm{~nm}$ in (N,O). (P-T) MitoTracker Red fluorescence of mitochondria in suspension cells of the WT (P), anti-1 (Q), anti-2 (R), and anti-3 (S). Bars, $10 \mu \mathrm{m}$. (T) Frequencies of the types of mitochondria in 200 suspension cells. Pu, punctiform; Ve, vermiform; Gi, giant; Di, diffuse.

smaller cells in MS medium (Fig. 3C-J). Of the three cell lines, anti-3 showed the smallest cells and the most seriously reduced growth (around 70\%) compared to the WT. Compared to the WT, the mitochondrial $\mathrm{H}_{2} \mathrm{O}_{2}$ content was increased in anti-1, anti-2, and anti-3, respectively (Fig. 3K and Supplemental Fig. 3). 
A
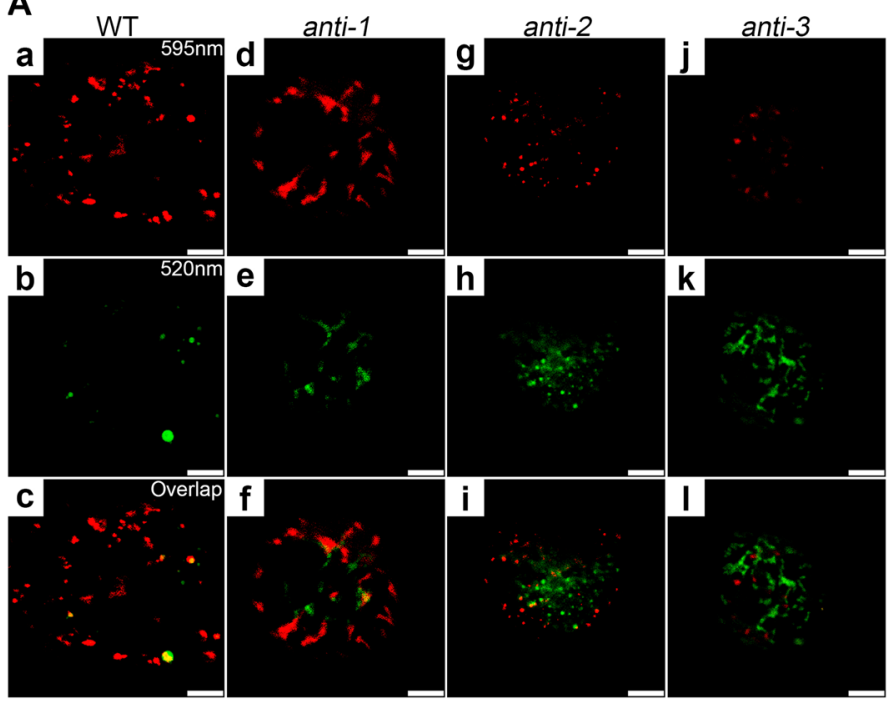

C
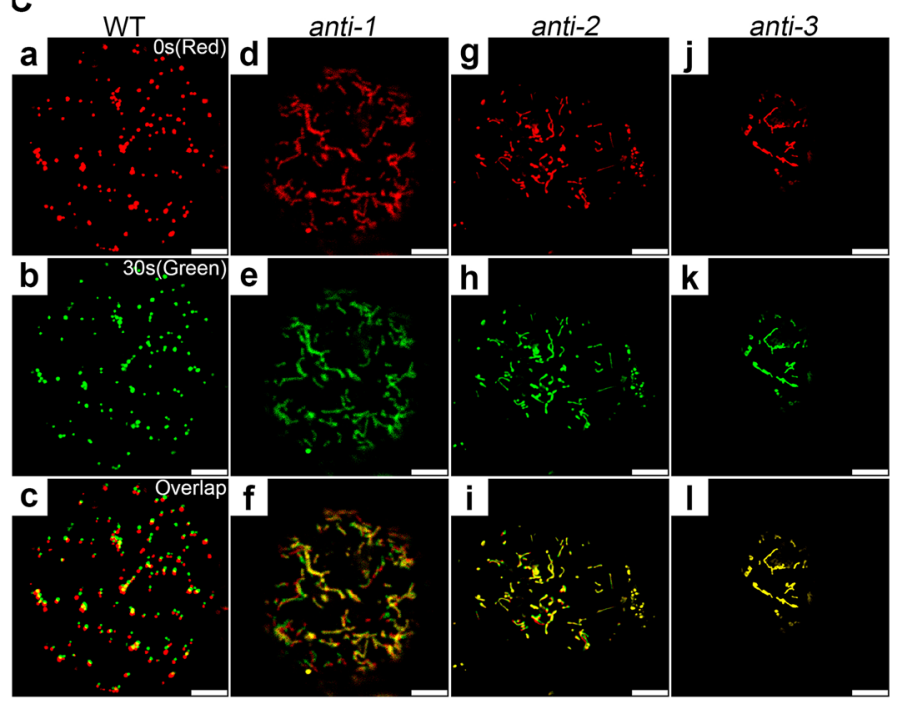

Figure 4. Mitochondrial $\Delta \psi \mathrm{m}$ in PtomtAPX-antisense suspension cells. (A) $\Delta \psi \mathrm{m}$ of mitochondria of suspension cells (JC-1 staining). Mitochondria with a high $\Delta \psi \mathrm{m}$ are shown in red (595 $\mathrm{nm}$ channel) (a), (d), (g) and (j); depolarized mitochondria are shown in green (520 nm channel); (b), (e), (h) and (k); (c), (f), (i) and (l), merged images; bars, $10 \mu \mathrm{m}$. (B) JC-1 red: green fluorescence ratios based on 200 cells. (C) CMXRos (red) fluorescence micrograph of mitochondrial mobility; green, pseudo-color; yellow, colocalized puncta; bars, 10 $\mu \mathrm{m}$. (D) ATP:ADP ratios. Bars, standard deviations. ** Significantly different at $\mathrm{P}<0.01$.

The structure of mitochondria in the PtomtAPX-antisense cells was assessed by transmission electron microscopy (TEM). WT cells exhibited a dense cytoplasm, clear structure, and a large vacuole occupying the majority of the cytoplasm (Fig. 3L). Numerous mitochondria with well-developed cristae and easily distinguishable outer and inner membranes were present in the WT cell lines (Fig. 3N). However, anti-1, anti-2, and anti-3 cells harbored a high frequency of abnormal mitochondria, which lacked cristae and internal structures, but retained a double membrane (Fig. 3M,O). According to CLSM, the proportion of mitochondria with an abnormal morphology was significantly increased in the transgenic cell lines, particularly in anti-3 (3.50\% normal mitochondria compared to $85.00 \%$ in the WT) (Fig. 3P-T).

Changes in mitochondrial morphology may influence their function and decrease their ATP-generating capacity. To assess mitochondrial dysfunction, the mitochondrial membrane potential $(\Delta \psi \mathrm{m})$ was determined using the fluorescent dye JC- 1 and by calculating the red:green fluorescence ratio ${ }^{27}$. The function of mitochondria was impaired and the $\Delta \psi \mathrm{m}$ was markedly decreased in the PtomtAPX-antisense cell lines (Fig. 4A,B). Next, we visualized mitochondrial movement by CMXRos staining. Mitochondria were selected in time-lapse videos at $0 \mathrm{~s}$ (red images) and $30 \mathrm{~s}$ (green images) and the two images were overlaid; yellow mitochondria were considered non-motile. Mitochondria in WT cells showed motility, and few were yellow. By contrast, mitochondria in anti- 1 and anti-2 cells showed reduced motility, while those in anti-3 cells showed little motility (Fig. 4C). Therefore, mitochondrial motility was markedly decreased in the PtomtAPX-antisense cells, suggesting impairment of mitochondrial function. 


\begin{tabular}{|l|l|l|l|}
\hline \multirow{2}{*}{ Group } & & \multicolumn{3}{|l|}{ nmol·min $^{-1} \cdot \mathbf{m g}^{-1}$ protein } \\
\cline { 2 - 4 } & Complex I & Complex II & Complex III \\
\hline WT & $93.59 \pm 11.46$ & $63.99 \pm 8.36$ & $99.28 \pm 9.17$ \\
\hline anti-1 & $75.71 \pm 8.14$ & $48.72 \pm 8.99$ & $81.17 \pm 6.59$ \\
\hline anti-2 & $58.60 \pm 13.91$ & $33.64 \pm 7.09$ & $52.75 \pm 11.07$ \\
\hline anti-3 & $31.71 \pm 11.18$ & $17.12 \pm 6.95$ & $25.32 \pm 9.58$ \\
\hline OX & $91.16 \pm 10.80$ & $62.43 \pm 8.12$ & $100.17 \pm 10.48$ \\
\hline
\end{tabular}

Table 2. Activity of respiratory complexes. Values are means \pm SDs of three replicates.

Because ATP is produced in mitochondria by oxidative phosphorylation, and the decreased mitochondrial $\Delta \psi \mathrm{m}$ may be due to inhibition of electron transport chain (ETC) complexes, the ATP:ADP ratio and mitochondrial complex I, II, and III activities were determined. Compared to the WT, the ATP:ADP ratio was about $65.52 \%, 29.47 \%, 14.81 \%$, and $21.27 \%$ in anti-1, anti-2, and anti-3 cells, respectively (Fig. 4D). Compared to the WT, complex I, II, and III activities were significantly decreased in anti-1, anti-2, and anti-3 cells, suggesting damage to ETC complexes (Table 2). Thus, energy production was decreased and ETC complexes were damaged in the PtomtAPX-antisense cells.

PCD is related to the overproduction of ROS in mitochondria ${ }^{22,28,29}$. Due to the reduced growth of antisense transgenic cell lines and plants, and the difficulty in obtaining the significant downregulation of the antisense plants, we suspected that deletion of PtomtAPX triggers PCD. We determined the degree of PCD in PtomtAPX-antisense suspension cell lines by two-channel flow cytometry (FCM). Normal (Q4, bottom left) and early apoptotic (Q3, bottom right) cells were stained by Hoechst dye, while apoptotic (Q1, top left) and advanced apoptotic (Q2, top right) cells were stained with propidium iodide (PI).

In the WT, most cells were normal $(>96 \%)$ and few were apoptotic $(<2 \%)$ or dead $(<2 \%)$. In comparison, the proportion of apoptotic anti-1, anti-2, and anti-3 cells was increased by $12.30 \%, 47.10 \%$, and $61.20 \%$, respectively(Fig. 5A-D). Thus, the increased $\mathrm{H}_{2} \mathrm{O}_{2}$ content in the PtomtAPX-antisense cell lines resulted in a significant increase in PCD.

To further assess the relationship between $\mathrm{H}_{2} \mathrm{O}_{2}$ content and $\mathrm{PCD}, 10 \mathrm{mM}$ exogenous $\mathrm{H}_{2} \mathrm{O}_{2}$ was added to the PtomtAPX-antisense cells to increase their $\mathrm{H}_{2} \mathrm{O}_{2}$ content. In the WT, adding $10 \mathrm{mM} \mathrm{H}_{2} \mathrm{O}_{2}$ increased the frequency of apoptotic cells by $>25 \%$, while the frequencies of apoptotic anti-1, anti-2, and anti-3 cells were increased by about $32.50 \%, 77.60 \%$, and $92.20 \%$, respectively(Fig. $5 \mathrm{E}-\mathrm{H}$ ). Treatment with $10 \mathrm{mM}$ exogenous $\mathrm{H}_{2} \mathrm{O}_{2}$ resulted in the death of almost all anti-3 cells. Therefore, exogenous $\mathrm{H}_{2} \mathrm{O}_{2}$ increased the frequency of PCD in the PtomtAPX-antisense cell lines. Therefore, the $\mathrm{H}_{2} \mathrm{O}_{2}$ content is related to the degree of PCD.

The above results show that decreased PtomtAPX expression is correlated with increased PCD, which may explain both the reduced growth and difficulty generating, Ptomt $A P X$-antisense $P$. tomentosa lines.

Mitochondrial dysfunction is due to an increased $\mathrm{H}_{2} \mathrm{O}_{2}$ content and oxidative damage. Non-enzymatic antioxidants such as ascorbate (AsA) and glutathione (GSH) can reduce the increased $\mathrm{H}_{2} \mathrm{O}_{2}$ level in mitochondria or the cytosol ${ }^{30}$. To determine the role of AsA and GSH in removing $\mathrm{H}_{2} \mathrm{O}_{2}$, we determined the mitochondrial AsA, DHA, GSH, and GSSG contents of the PtomtAPX-antisense cell lines. Compared to the WT, the AsA contents were $52.07 \%, 37.43 \%$, and $15.70 \%$, and the AsA:DHA ratios were $48.56 \%, 34.08 \%$, and $14.52 \%$ in mitochondria of anti-1, anti-2, and anti-3 cells, respectively (Supplemental Fig. 4A). Compared to the WT, the GSH contents were $51.51 \%, 40.79 \%$, and $27.37 \%$, and the GSH:GSSG ratios were $43.40 \%, 36.01 \%$, and $22.22 \%$, in mitochondria of anti-1, anti-2, and anti-3 cells, respectively (Supplemental Fig. 4B). The greatest decreases in the AsA:DHA and GSH:GSSG ratios occurred in anti-3 cells. Thus, the transgenic cells are under oxidative stress and their $\mathrm{H}_{2} \mathrm{O}_{2}$ content could not be balanced by AsA and GSH.

An increased $\mathrm{H}_{2} \mathrm{O}_{2}$ content may lead to oxidative damage and reduce mitochondrial efficiency. Therefore, we evaluated mitochondrial lipid peroxidation by quantifying malondialdehyde (MDA) levels. Compared to the WT, the mitochondrial lipid peroxidation level was 1.74-, 2.55-, and 3.65-fold higher in anti-1, anti-2, and anti3, respectively (Supplemental Fig. 4C). Moreover, the levels of oxidized proteins (with carbonyl groups) were increased in anti-1, anti-2, and anti-3, respectively, as determined by Western blotting (Supplemental Fig. 4D).

Therefore, the decreased PtomtAPX protein levels in the PtomtAPX-antisense cells resulted in increased $\mathrm{H}_{2} \mathrm{O}_{2}$ content, which could not be balanced by enzymatic or non-enzymatic antioxidant mechanisms due to the structural and functional impairment of mitochondria.

Overexpression of PtomtAPX decreases $\mathrm{H}_{2} \mathrm{O}_{2}$ content and slows growth. PtomtAPX-overexpressed cell lines were generated using a PtomtAPX overexpression construct under the control of the $35 \mathrm{~S}$ promoter (35Spro:PtomtAPX) (Fig. 6). qRT-PCR and ELISA showed that the PtomtAPX transcript and protein levels were increased 4-fold and 2.7-fold in the Ptomt APX-overexpressed cell lines compared to the WT, respectively.

For growth in MS medium, the PtomtAPX-overexpressed cell lines required $0.2 \mathrm{mg} / \mathrm{L} 6$-BA. Compared to the WT, the PtomtAPX-overexpressed cell lines showed slowed growth and an abnormal morphology. The structure of mitochondria in $O X$ cells was also abnormal by TEM and CLSM. The mitochondrial $\Delta \psi \mathrm{m}$, motility, and energy production (ATP:ADP ratio) were decreased in the PtomtAPX-overexpressed plants, suggesting impairment of mitochondrial function. However, the complex I, II, and III activities were unchanged in OX (Table 2), suggesting that the respiratory chain complexes were not damaged. 

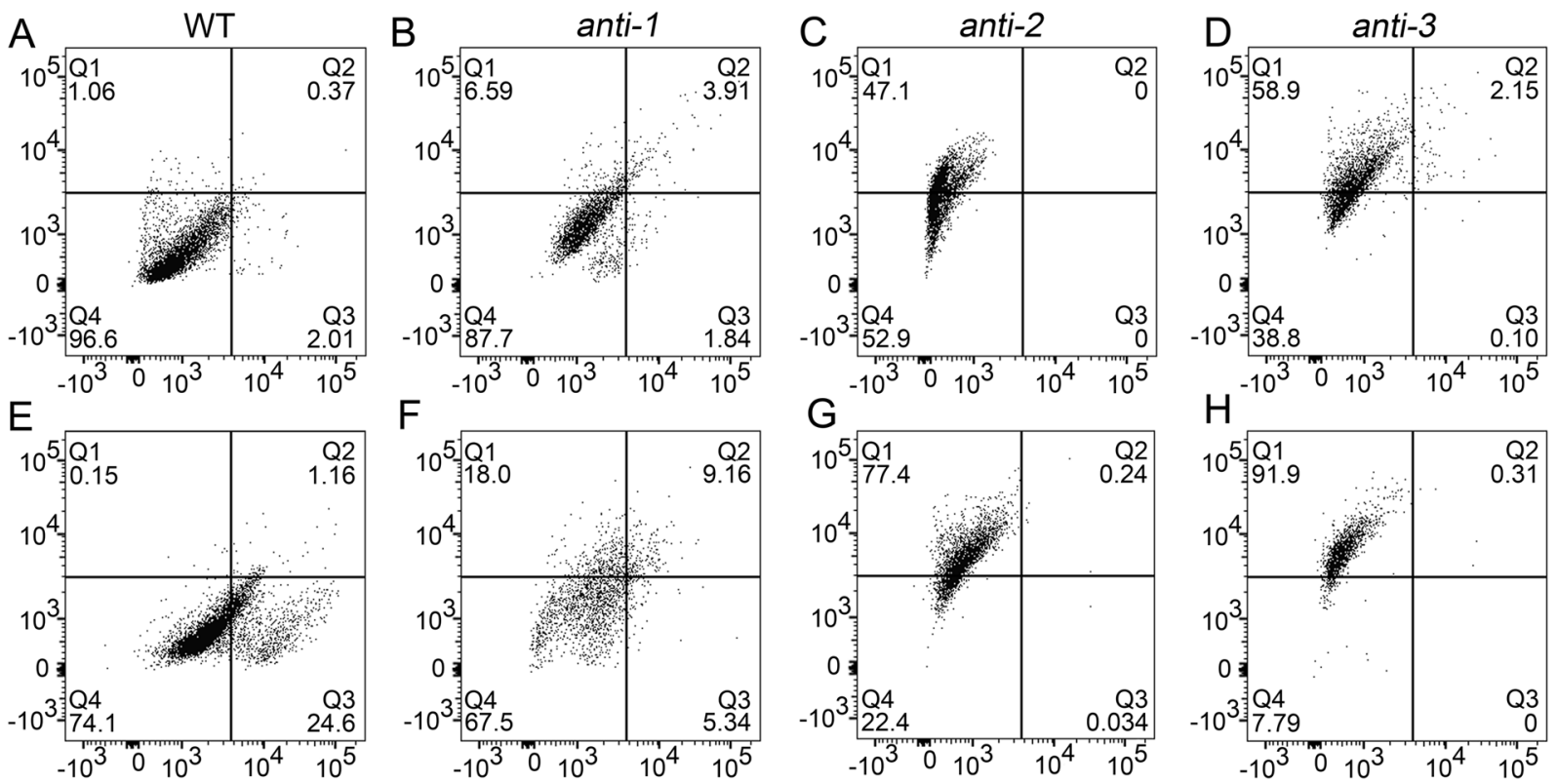

Figure 5. $P C D$ of $P$ tomt $A P X$-antisense suspension cells. (A,D,G,J) $P C D$ under untreated conditions. (B,E, H,K) PCD under $\mathrm{H}_{2} \mathrm{O}_{2}$ treatment $(10 \mathrm{mM}, 3 \mathrm{~h}$ ). Frequencies were calculated based on 5,000 cells per treatment.

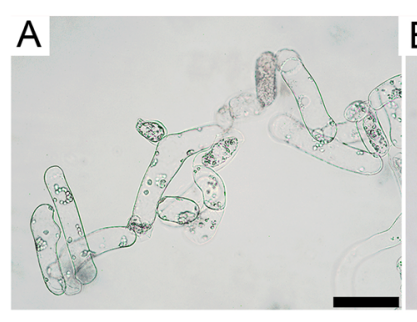

B
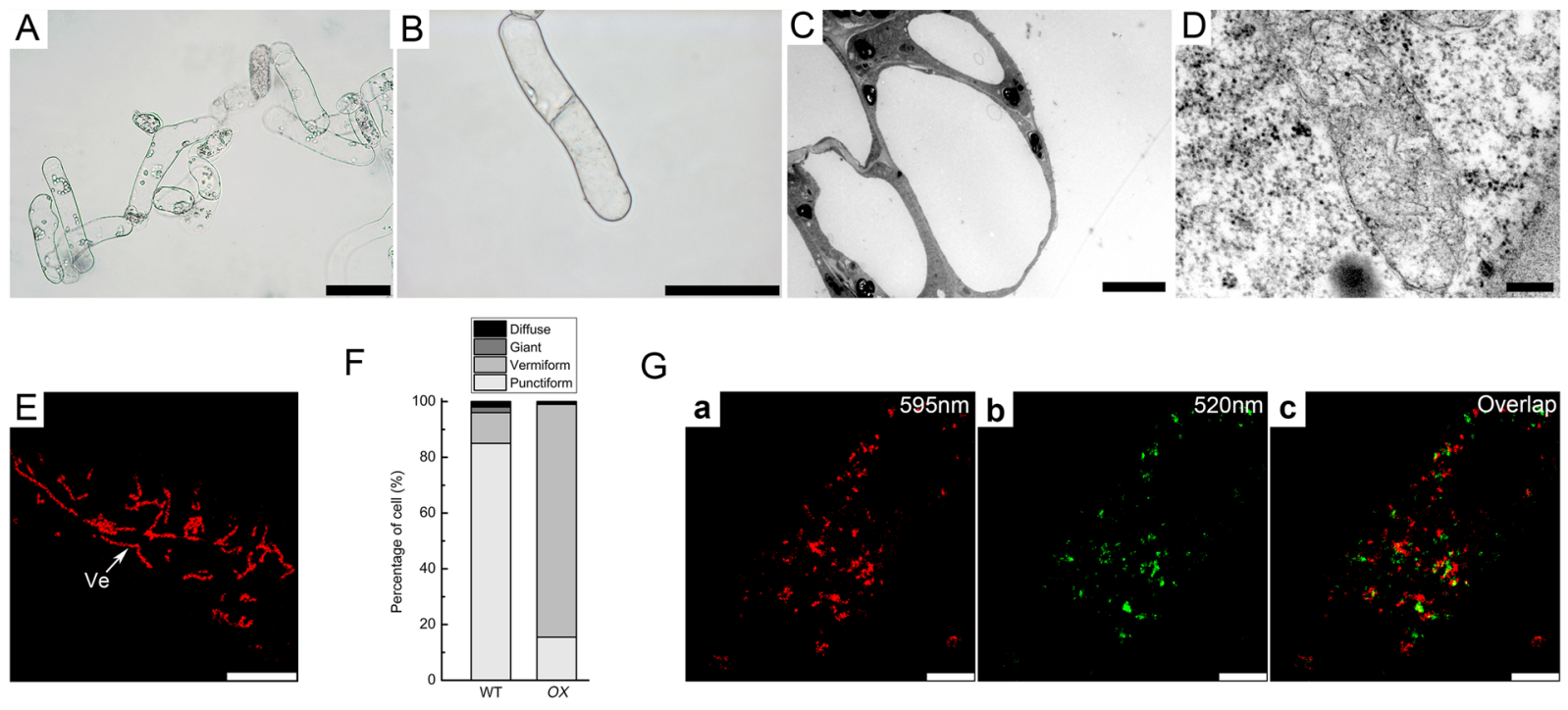

G
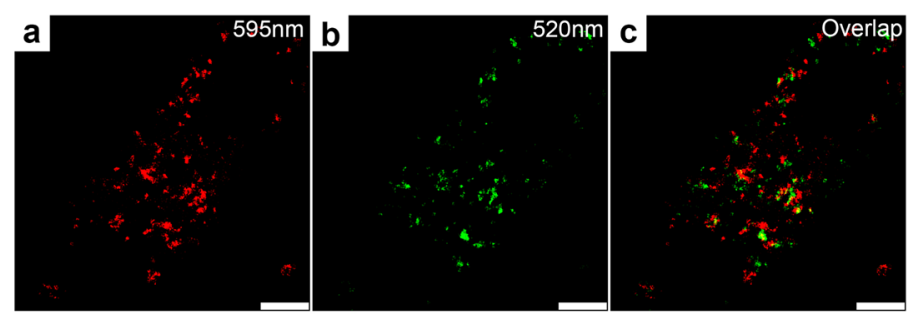

$\mathrm{H}$
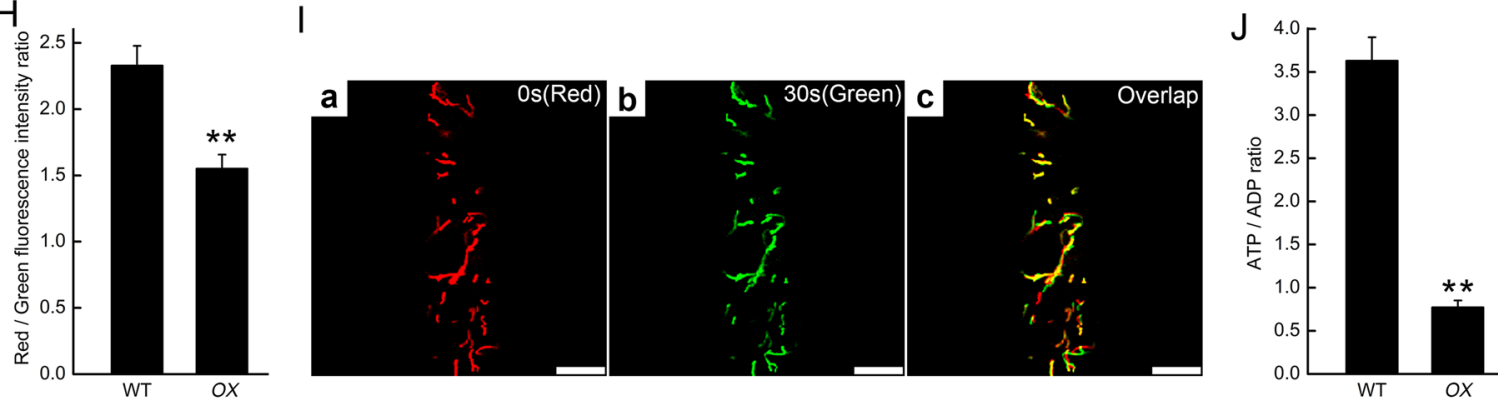

Figure 6. Growth reduction and mitochondrial dysfunction in PtomtAPX-overexpressed cells. $(\mathbf{A}, \mathbf{B})$ Morphology of PtomtAPX-overexpressed cells; bars, $100 \mu \mathrm{m}$. (C,D) Morphology of cells and mitochondria according to TEM; bars, $5 \mu \mathrm{m}$ in (C) and $200 \mathrm{~nm}$ in (D). (E,F) MitoTracker Red fluorescence of mitochondria; bars, $10 \mu \mathrm{m}$; Frequencies were calculated based on 200 cells. Ve, vermiform. (G) $\Delta \psi \mathrm{m}$ of mitochondria (JC-1 staining). (H) JC-1 polymer (red):monomer (green) fluorescence ratios based on 200 cells. WT, WT cells; OX, PtomtAPX-overexpressed cells. (I) Mitochondrial mobility; bars, $10 \mu \mathrm{m}$. (J) ATP:ADP ratios. **Significantly different at $\mathrm{P}<0.01$. Bars, standard deviations. 
The mitochondrial $\mathrm{H}_{2} \mathrm{O}_{2}$, AsA and GSH contents and AsA/DHA and GSH/GSSG ratios of the PtomtAPX-overexpression cells were not significantly different from those of the WT (Supplemental Figs. 4 and 5). The mitochondrial MDA level in PtomtAPX-overexpression cells was $41.02 \%$ of the WT (Supplemental Fig. 5). The oxidized protein level in PtomtAPX-overexpressed cells was slightly lower than that of the WT (Supplemental Fig. 4). These results suggest that oxidative damage was alleviated in the PtomtAPX-overexpression cells compared to the WT.

We suspected that the decreased $\mathrm{H}_{2} \mathrm{O}_{2}$ content in $\mathrm{OX}$ caused mitochondrial dysfunction, because $\mathrm{H}_{2} \mathrm{O}_{2}$ is an important signaling molecule. To further assess the relationship between $\mathrm{H}_{2} \mathrm{O}_{2}$ content and mitochondrial dysfunction, exogenous $\mathrm{H}_{2} \mathrm{O}_{2}$ was added to PtomtAPX-overexpressed cells. Addition of 10 or $100 \mathrm{mM}$ exogenous $\mathrm{H}_{2} \mathrm{O}_{2}$ to PtomtAPX-overexpressed cells significantly alleviated mitochondrial damage and partially restored the mitochondrial membrane potential (Supplemental Fig. 5). Therefore, overexpression of PtomtAPX resulted in a decreased $\mathrm{H}_{2} \mathrm{O}_{2}$ content and mitochondrial dysfunction.

Gene expression in PtomtAPX-antisense and PtomtAPX-overexpressed cell lines. To further characterize the effects of PtomtAPX on mitochondria, we performed RNA-seq analyses of WT and PtomtAPX-antisense cells. Compared to the WT, 3,571 genes with significantly different expression levels (fold change $>1.5$ or $<-1.5$, and corrected $\mathrm{P}<0.001$ ) were detected in anti-3. (Fig. 7A and Supplemental Dataset 1). This indicates that oxidative damage is the cause of the significantly differential gene expression in anti-3.

We clustered genes with similar expression patterns to further investigate the dynamic trend of the normalization of gene expression. The 3,571 genes were grouped into six clusters (Fig. 7B and Supplemental Dataset 1). Genes in cluster 1 ( 2 genes) and cluster 2 (781 genes) were downregulated, and the genes in cluster 4 (18 genes) and cluster 5 (2,612 genes) were upregulated in anti-3, and were wholly or partly normalized in anti-3-A, indicating that they are directly related to the abnormal morphology of cells and mitochondria. The expression levels of genes in cluster 3 ( 83 genes) which were downregulated in anti-3 and cluster 6 ( 75 genes) but upregulated in anti-3 were not normalized in anti-3-A (Fig. 7B and Supplemental Dataset 1).

Gene Ontology (GO) term enrichment analyses were performed to further characterize the function of the differentially expressed genes in anti-3 (Supplemental Dataset 2). The GO term "integral component of membrane" $(\mathrm{P}<0.005)$ "UDP-glycosyltransferase activity" and "protein phosphorylation" $(\mathrm{P}<0.005)$ showed enrichment (Supplemental Dataset 2). In particular, an OXPHOS-related gene (phosphatase family protein, POPTR_0007s09470) and inorganic pyrophosphatase (POPTR_0005s01840) were significantly downregulated in anti-3 (Fig. 7C). The activity of inorganic pyrophosphatase, which hydrolyzes inorganic pyrophosphate into two phosphates, is essential for many biosynthetic reactions and energy metabolism as well as for maintaining mitochondrial function. The deletion of inorganic pyrophosphatase compromises cell viability due to the loss of mitochondrial function ${ }^{31}$. The decreased expression of those genes in anti-3 was consistent with the damaged respiratory ETC in mitochondria of anti-3. The gene encoding phosphatidylinositol-4-phosphate 5-kinase (POPTR_0005s15850), which is involved in cytoskeletal rearrangement ${ }^{32}$, was also downregulated in anti-3 (Fig. 7C). A cytokinin riboside 5'-monophosphate phosphoribohydrolase gene (POPTR_0004s22210), the product of which activates cytokinins ${ }^{33}$, and a set of UDP-glycosyltransferases (POPTR_0001s05400, POPTR_0006s02390, POPTR_0545s00210 and POPTR_0009s15190), which are related to mitosis and strongly induced in dividing cells ${ }^{34}$, were downregulated in anti-3 (Fig. 7C). Cytokinins are master plant hormones that control cell division, senescence, and growth ${ }^{35}$. The decreased expression of those genes in anti- 3 is consistent with the reduced proliferation of transgenic cells. In addition, a gene related to leaf senescence, alkaline $\alpha$-galactosidase (POPTR_0016s05500) ${ }^{36}$, was downregulated in anti-3 (Fig. 7C). These genes are implicated in the altered growth and morphology of transgenic cells, which were smaller and had a slower growth rate than WT cells.

RNA-seq analyses of WT and PtomtAPX-overexpressed cells treated or not with $10 \mathrm{mM} \mathrm{H}_{2} \mathrm{O}_{2}$ were performed. Compared to the WT, 5,542 genes with significantly different expression levels (fold change $>1.5$ or $<-1.5$, and corrected $\mathrm{P}<0.001)$ were detected in $O X$. The expression of $66.47 \%(3,684$ genes) of those genes was normalized by $10 \mathrm{mM} \mathrm{H}_{2} \mathrm{O}_{2}(\mathrm{OX}-\mathrm{H})$ (Fig. $7 \mathrm{D}$ and Supplemental Dataset 1$)$. Thus, the decreased $\mathrm{H}_{2} \mathrm{O}_{2}$ content caused the significantly different gene expression levels in $O X$.

We also clustered genes with similar expression profiles to investigate the trends of these RNAs in OX compared to the WT. As shown in Fig. 7E, the 5,542 genes were grouped into six clusters (Supplemental Dataset 1). Genes in cluster 1 ( 1 genes) and cluster 2 (1,486 genes) were downregulated, and those in cluster 4 (127 genes) and cluster 5 (2,070 genes) were upregulated in $O X$ and were normalized in $\mathrm{OX}-\mathrm{H}$, indicating that they are directly related to the abnormal morphology of cells and mitochondria. Genes in cluster 3 (643 genes), which were downregulated in $O X$, and cluster 6 (1,215 genes), which were upregulated in $O X-\mathrm{H}$, were not normalized in $O X-\mathrm{H}$ (Fig. 7E and Supplemental Dataset 1). The GO term "hydrolase activity, acting on ester bonds" was significantly enriched (Supplemental Dataset 2). For example, two phosphatases (POPTR_0014s08130 and POPTR_0013s14030) were downregulated in $O X$ and recovered to normal expression level in $O X-\mathrm{H}$ (Fig. 9F). Moreover, two cytokinin riboside 5'-monophosphate phosphoribohydrolases (POPTR_0016s09180 and POPTR_0009s01600), an UDP-glycosyltransferase (POPTR_0003s14230), and a phosphatidylinositol-4-phosphate 5-kinase (POPTR_0005s15850) were significantly differentially expressed in OX, and their expression was normalized in $O X-\mathrm{H}$ (Fig. 7F).The genes in cluster 5 were upregulated in $O X$ and normalized in $O X-\mathrm{H}$. For example, inorganic pyrophosphatase (POPTR_0006s08250) impairs mitochondrial function and was normalized in OX-H (Fig. 7F). These genes may be related to growth reduction, cell morphology and mitochondrial dysfunction due to their important roles in mitochondrial function, cytoskeleton, cytokinin activation, and cell division ${ }^{31-34}$. This is consistent with the need for the addition of 6-BA for the growth of PtomtAPX-overexpressed cells and the normalization of cellular morphology and growth rate, and mitochondrial function, by exogenous $\mathrm{H}_{2} \mathrm{O}_{2}$. 
A

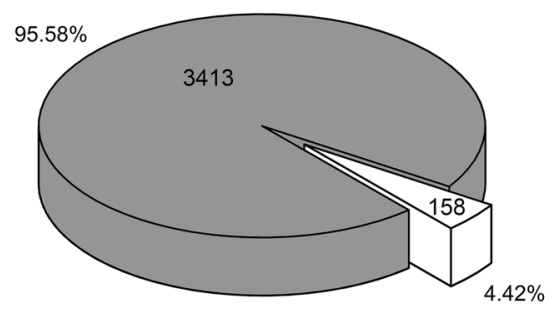

C

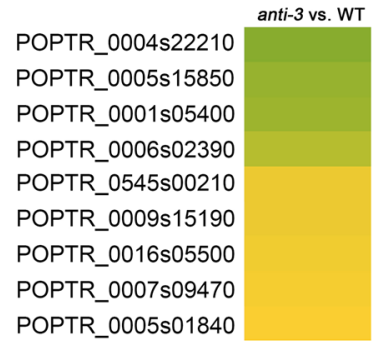

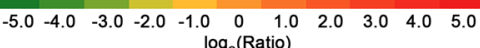

B

$\log _{2}$ (Ratio)

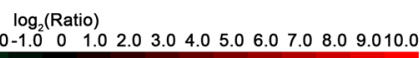

anti-3

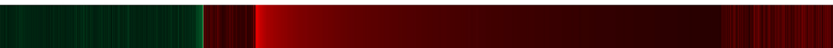

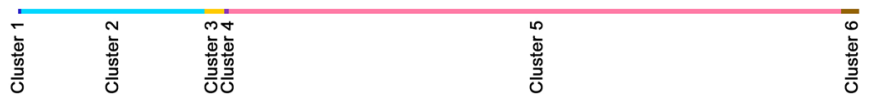

D

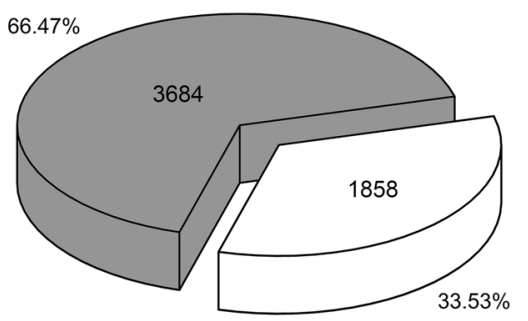

$\mathbf{F}$

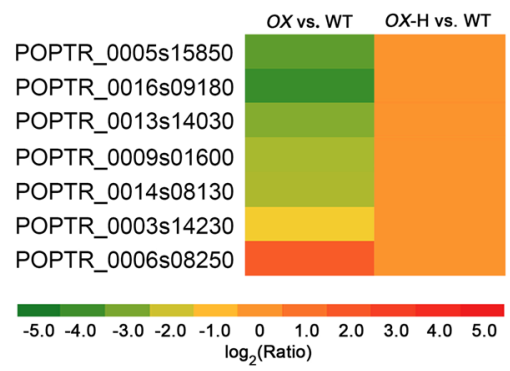

E

$-10.0-9.0-8.0-7.0-6.0-5.0-4.0-3.0-2.0-1.0 \quad 0 \quad 1.02 .03 .04 .05 .06 .07 .0 \quad 8.09 .010 .0$

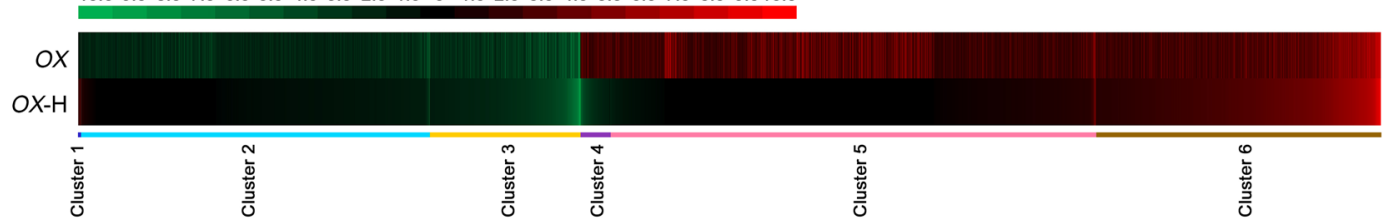

Figure 7. RNA-seq analyses of anti-3 and OX. (A) DEGs of anti-3 (anti-3 vs. WT) and their expression under AsA treatment $(1 \mathrm{mM}, 3 \mathrm{~h}$ ) (corrected P-value $<0.001$, fold change $>1.5$ or $<-1.5$; gray, genes recovered to normal expression level under AsA treatment). (B) Clustering of expression ratios (anti-3 vs. WT). Red, upregulation; green, downregulation. (C) Heat map of genes related to phenotypes of cells and mitochondria in anti-3, based on the RPKM values. (D) DEGs of $O X\left(O X\right.$ vs. WT) and their expression under $\mathrm{H}_{2} \mathrm{O}_{2}$ treatment $(10 \mathrm{mM}, 3 \mathrm{~h}$ ) (corrected $\mathrm{P}$-value $<0.001$, fold change $>1.5$ or $<-1.5$; gray, genes recovered to normal expression level under $\mathrm{H}_{2} \mathrm{O}_{2}$ treatment). (E) Clustering displays of expression ratios (OX vs. WT). Red, upregulation; green, downregulation. (F) Heat map of genes related to phenotypes of cells and mitochondria in $O X$, based on RPKM values.

Non-enzymatic and enzymatic mechanisms maintain redox homeostasis in plant cells. $\mathrm{H}_{2} \mathrm{O}_{2}$ is scavenged principally by SOD, CAT, GPX, and PrxR, and the AsA-GSH cycle. None of these ROS-scavenging enzymes was significantly differentially expressed in anti-3 or OX compared to the WT, suggesting that the ROS pathway is not induced by the downregulation or upregulation of PtomtAPX.

APX mediates cross-compartment protection against the deleterious effects of $\mathrm{H}_{2} \mathrm{O}_{2}{ }^{37}$. To determine whether APXs in other compartments can compensate for the loss of mitochondrial APX, we evaluated the expression levels of APXs in the chloroplasts, cytoplasm, and microbody. None of the APX genes was significantly differentially expressed in the PtomtAPX-antisense and PtomtAPX-overexpressed cell lines, suggesting that mitochondrial PtomtAPX is independent of APXs in other compartments and so provides little cross-compartment protection against $\mathrm{H}_{2} \mathrm{O}_{2}$.

Taken together, these results suggest that PtomtAPX expression is closely associated with $\mathrm{H}_{2} \mathrm{O}_{2}$ content and mitochondrial structure. Moreover, appropriate PtomtAPX expression is necessary to maintain a mitochondrial $\mathrm{H}_{2} \mathrm{O}_{2}$ level appropriate for mitochondrial structure and function in P. tomentosa. 


\section{Discussion}

Plant mitochondria are major organelles of ROS production and are targets of ROS ${ }^{38,39}$. ROS-scavenging systems control the ROS balance in plant mitochondria. We identified two APXs, PtomtAPX and PtosAPX, in the mitochondria of $P$. tomentosa. PtomtAPX is specifically targeted to mitochondria and is the major APX involved in controlling mitochondrial $\mathrm{H}_{2} \mathrm{O}_{2}$ levels, and it influences mitochondrial structure and function and PCD in P. tomentosa. PtosAPX is targeted to both mitochondria and chloroplasts and is the minor role in controlling mitochondrial $\mathrm{H}_{2} \mathrm{O}_{2}$ levels.

Targeting peptides of mitochondrial and chloroplastic proteins (mTPs, also termed pre-sequences and cTPs, also termed transit peptides) has a similar amino acid composition and are indistinguishable by sequence analy$\operatorname{ses}^{40,41}$. It is possible that mitochondrial isoforms have been misidentified as chloroplastic isoforms. For example, there were four chloroplastic APX in rice, but no mitochondrial isoform was confirmed ${ }^{2,5,6}$, indicating that mitochondrial isoform was mistaken for chloroplastic iosform. In this study, PtomtAPX and PtosAPX of P. tomentosa were also marked as chloroplastic isoforms (Supplemental Fig. 2). However, plant mTPs and cTPs exhibit quantitative and structural differences ${ }^{42}$.

Positively charged residues and amphipathic $\alpha$-helix formed in $19 \mathrm{~N}$-terminal amino acid are responsible for localization ${ }^{26,42,43}$. Our results indicate that chloroplastic APX has high serine and proline contents and a low arginine content. By contrast, PtomtAPX has three arginine residues and two amphipathic $\alpha$-helices in its $19 \mathrm{~N}$-terminal portion of targeting peptide (Fig. 1A). A rice chloroplastic APX isoform, OsAPX6, has been found in mitochondria of BY-2 tobacco but its presence in chloroplasts is unclear ${ }^{2,6,44}$. We found that the mTPs of OsAPX5 and OsAPX6 contained four positively charged amino acids (three of them are arginine residues) and an amphipathic $\alpha$-helix, suggesting that they are targeted to mitochondria (Fig. 1A). Furthermore, three positively charged amino acids (two of them are arginine residues) and an amphipathic $\alpha$-helix are present in Z. mays APX7 (Fig. 1A). Therefore, mitochondrial APX isoforms are present in various plant species, including model plants such as rice. The results of comparing the amino acid composition of targeting peptide of at least 100 proteins from diverse plant species showed increased intermediate amino acid led to the targeted of both chloroplasts and mitochondria ${ }^{44-48}$. Targeting of the same protein to two locations can result in co-regulation without a change in the genome. The presence of co-targeted enzymes of the AsA-GSH cycle may have a beneficial effect by restoring a normal level of ROS, which is important for the function of mitochondria and plastids.

Plant mitochondria in photosynthetic and non-photosynthetic organs have defense systems to prevent damage by $\mathrm{H}_{2} \mathrm{O}_{2}$ and to respond to environmental stresses ${ }^{49-51}$. Our results indicate that $P$. tomentos $a$ has at least two mitochondrial APXs, PtomtAPX and PtosAPX, which have similar levels of activity. However, mitochondria had a low PtosAPX level, suggesting that PtosAPX is not an important player in ROS scavenging in mitochondria. The PtosAPX level in mitochondria was markedly lower than that of PtomtAPX, while the PtosAPX level in chloroplasts was higher than that in mitochondria, suggesting that PtomtAPX and PtosAPX are mitochondrial and chloroplastic isoforms, respectively. This is consistent with a previous report that dual-targeted proteins are more strongly targeted to one of the two organelles ${ }^{44}$.

The expression of PtomtAPX and PtosAPX differed significantly in the presence of various environmental stresses. In this study, the expression of PtosAPX and PtotAPX increased by high-light stress, whereas that of PtomtAPX was only slightly affected. The expression of chloroplastic APX(chlAPX) varies according to light intensity; e.g., high-intensity light decreases the expression of chlAPX in spinach leaves ${ }^{52}$. Exposure of Arabidopsis lacking tAPX to high-intensity light and MV stresses result in increased $\mathrm{H}_{2} \mathrm{O}_{2}$ accumulation and oxidation of proteins ${ }^{53}$. A mutant wheat line with decreased tAPX activity shows reduced photosynthetic activity and biomass accumulation in the presence of high-intensity light, suggesting that tAPX is essential for photosynthesis. Therefore, PtosAPX plays a role in the photosynthetic apparatus and PtomtAPX functions in the non-photosynthetic apparatus.

The major $\mathrm{H}_{2} \mathrm{O}_{2}$ detoxifying system in plant cells is the AsA-GSH cycle, in which APX is the key enzyme ${ }^{49,50}$. However, stromal/mitochondrial APX-knockdown lines, as well as single and double null mutants in chlAPX (sAPX and tAPX) in A. thaliana and rice, exhibit a normal phenotype and normal biochemical and physiological attributes under normal growth conditions, suggesting that chloroplast stromal/mitochondrial APX is not important for $\mathrm{H}_{2} \mathrm{O}_{2}$ scavenging in A. thaliana or rice ${ }^{23,24}$. In our research, the PtomtAPX-antisense cells under normal growth conditions had significantly higher $\mathrm{H}_{2} \mathrm{O}_{2}$ levels and an abnormal phenotype, including impaired mitochondrial structure and function and slowed growth. Transcriptomic analyses of anti-3 and WT revealed that the expression levels of genes encoding phosphatases and inorganic pyrophosphatases were significantly different, while genes related to cell growth (such as cytoskeleton, cytokinin, cell division, and senescence) were downregulated in anti-3. PtosAPX, was not upregulated in anti-3, indicating that PtosAPX cannot compensate for the function of PtomtAPX. Therefore, PtomtAPX, not PtosAPX, is the major APX in mitochondria.

Glutathione acts as a mobile pool of non-protein reduced sulfur, as an antioxidant, and in the detoxification of xenobiotics and heavy metals ${ }^{1,30}$. The GSH:GSSG ratio is an important indicator of the redox balance in plant cells. GR plays a central role in maintaining the GSH pool during stress ${ }^{1}$. The transcript level of $G R$, which plays a central role in maintaining the GSH pool, was not significantly increased in the PtomtAPX-antisense cells, and the GSH/GSSG ratio was significantly decreased, suggesting an insufficient glutathione pool to counteract the $\mathrm{H}_{2} \mathrm{O}_{2}$ level. Moreover, none of the APXs were significantly differentially expressed in anti-3 or OX according to RNA-seq analyses, indicating that cross-compartment protection among different organelles is not enough to protect mitochondria in the absence of PtomtAPX. Therefore, PtomtAPX is required to modulate $\mathrm{H}_{2} \mathrm{O}_{2}$ levels in mitochondria.

Over-accumulation of ROS triggers plant cell death. Excess ROS alter mitochondrial membrane permeability, decrease $\Delta \Psi \mathrm{m}$, reduce the electron density and number of cristae, and compromise inner and outer membranes; these effects trigger the release of cytochrome c, resulting in PCD. In plants, PCD is activated during various developmental processes and under diverse stress conditions ${ }^{54,55}$. Our results indicate that PCD was 
triggered by increased $\mathrm{H}_{2} \mathrm{O}_{2}$ levels and decreased PtomtAPX expression in the PtomtAPX-antisense cells. The addition of exogenous $\mathrm{H}_{2} \mathrm{O}_{2}$ to the PtomtAPX-antisense cells resulted in increased PCD. This is consistent with the slowed plant growth, small cells, and mitochondrial aggregation, swelling, loss of electron density and cristae, and decrease in $\triangle \Psi \mathrm{m}$ in the Ptomt $A P X$-antisense lines. Thus, downregulation of PtomtAPX expression in the PtomtAPX-antisense plants induced PCD and slowed growth. This may explain the difficulty generating PtomtAPX-antisense plants under normal growth conditions.

The decreased levels of PtomtAPX protein in the PtomtAPX-antisense cells resulted in higher $\mathrm{H}_{2} \mathrm{O}_{2}$ levels, which could not be counteracted by enzymatic or non-enzymatic mechanisms due to damaged and dysfunctional mitochondria. ROS can damage, by indiscriminate oxidation of macromolecules, various cellular compartments. In this study, excessive $\mathrm{H}_{2} \mathrm{O}_{2}$ levels impaired mitochondrial function in the transgenic lines. This is consistent with a previous report that high levels of ROS oxidize mitochondrial structural proteins, which reduces the efficiency of OXPHOS ${ }^{56}$. The damage to mitochondria was significantly alleviated by the addition of exogenous AsA.

The decreased $\mathrm{H}_{2} \mathrm{O}_{2}$ levels in the PtomtAPX-overexpressed cells resulted in decreased membrane potential and ATP production. However, mitochondrial lipids and structural proteins, specifically those related to OXPHOS, were not oxidized, suggesting that maintenance of ROS homeostasis is necessary for OXPHOS in the PtomtAPX-overexpressed cells. Moreover, RNA-seq showed that the expression of genes related to mitochondrial function, cytoskeleton, cytokinin activation, and cell division in $O X$ cells was significantly recovered by the addition of exogenous $\mathrm{H}_{2} \mathrm{O}_{2}$. Thus, low $\mathrm{H}_{2} \mathrm{O}_{2}$ levels also impair mitochondrial function by an unknown mechanism.

ROS as signaling molecules regulate a number of processes during plant growth and development, such as cell elongation and differentiation, as well as the responses to a variety of environmental stimuli ${ }^{56,57}$. For example, in soybean, $\mathrm{H}_{2} \mathrm{O}_{2}$ levels increase from the tip of the hypocotyl (elongation zone) to the highly lignified base. In onion root fragments, $\mathrm{H}_{2} \mathrm{O}_{2}$ levels are high in cells undergoing elongation and lignification, and the former have lower total peroxidase activity ${ }^{58}$. Therefore, maintaining appropriate $\mathrm{H}_{2} \mathrm{O}_{2}$ levels is important for mitochondrial efficiency and cell growth in $P$. tomentosa, although the mechanism involved is unknown.

In conclusion, PtomtAPX is specifically targeted to mitochondria in which it is the primary APX, while PtosAPX is dual-targeted to mitochondria and chloroplasts. Expression of PtomtAPX in mitochondria is necessary for regulation of mitochondrial ROS levels and maintenance of mitochondrial function in P. tomentosa. Our findings provide valuable insight into the mechanism by which ROS balance is maintained and the function of the AsA-GSH cycle in mitochondria.

\section{Materials and Methods}

Plant materials and growth conditions. Suspension cells of $P$. tomentosa Carr. were grown in the dark at $25^{\circ} \mathrm{C}$. Transgenic suspension cells were obtained according to Li et al..$^{59}$.

Molecular cloning and plasmid construction. Full-length PtomtAPX and PtosAPX cDNA were amplified using the primers PtomtAPX-F/R and PtosAPX-F/R, respectively, and sequenced. PtomtAPX and PtosAPX was amplified using G-Ptomt $A P X-F / R$ and $G-P t o s A P X-F / R$ and cloned into the $\mathrm{pBI} 121-\mathrm{GFP}$ vector to generate 35S:PtomtAPX-GFP and 35S:PtosAPX-GFP, respectively. Full-length PtomtAPX cDNA was amplified using $O X-P$ tomtAPX-F/R and cloned into pBI121 to generate 35S:PtomtAPX (Supplemental Table 1). Full-length PtomtAPX cDNA was amplified using anti-PtomtAPX-F/R and cloned into pBI121. The amphiphilic $\alpha$-helice is predicted using Bioedit 7.0.

Enzyme-activity assays. The ORF minus the targeting peptide of PtomtAPX and PtosAPX was amplified by PCR using $P$-Ptomt APX-F/R and P-PtostAPX-F/R, respectively, and inserted downstream of the pET30a plasmid T7 promoter (Novagen, Madison, WI). PtomtAPX and PtosAPX were expressed and purified according to Zhang et al. ${ }^{60}$. The activity of purified recombinant PtomtAPX using ASA and $\mathrm{H}_{2} \mathrm{O}_{2}$ as substrates was assayed as described previously ${ }^{59}$.

PtomtAPX and PtosAPX immunolocalization. Purified recombinant PtomtAPX or PtosAPX (5 mg) was used to immunize rabbits at 3-week intervals. The specificity of the anti-PtomtAPX and anti-PtosAPX antibodies was confirmed by hybridization with a membrane blotted with protein extracts from leaves of $P$. tomentosa. P. tomentosa leaves were fixed and subjected to immunolocalization as described previously ${ }^{61}$.

PtomtAPX-GFP and PtosAPX-GFP fusion targeting analyses. The GFP gene was amplified by PCR using the primers GFP-F/GFP-R. The product was inserted into pBI121(Clontech), which lacked the GUS-coding region to create the GFP(A)-pBI121 vector, the fusion construct of 35S:PtomtAPX-GFP, and 35S:PtomtAPX-GFP. Transgenic tobacco leaf epidermis and root-tip cells were visualized by CLSM (Leica SP8).

Enzyme-linked immunosorbent assays (ELISA). An anti-PtomtAPX or anti-PtosAPX antibody $(1: 5,000$ in PBS) was added to each well of a microtiter plate, followed by a goat anti-rabbit IgG HRP-conjugated secondary antibody (1:10,000 in PBS) (Supplemental Fig. 6). Protein from leaves or suspension cells was used for ELISA in accordance with the method of Zhang et al. ${ }^{61}$. Optical density values were determined using a Bio-Rad 680 Microplate Reader.

qRT-PCR. $\quad$ qRT-PCR was performed with the $q R T-P$ tomt $A P X-F / R$ or qRT-PtosAPX primers. The PCR conditions were $94^{\circ} \mathrm{C}$ for $5 \mathrm{~min} ; 40$ cycles at $94^{\circ} \mathrm{C}$ for $20 \mathrm{~s}, 58^{\circ} \mathrm{C}$ for $20 \mathrm{~s}, 72^{\circ} \mathrm{C}$ for $30 \mathrm{~s}$, and $60^{\circ} \mathrm{C}$ for $30 \mathrm{~s}$; and $72^{\circ} \mathrm{C}$ for $1 \mathrm{~min}$. qRT-actin-F/R was used as normalization. Data were calculated using the $2^{-\Delta \Delta \mathrm{Ct}}$ method.

TEM. Suspension cells were fixed and embedded as described by Zhang et al. ${ }^{61}$. 
CLSM. CLSM was performed using a Leica TCS-SP8 CLSM. The JC-1 signal was visualized by excitation at $488 \mathrm{~nm}$ and emission at 520-540 nm (green fluorescence) and 595-625 nm (red fluorescence). MitoTracker Red CMXRos (Invitrogen) was visualized by excitation at $578 \mathrm{~nm}$ and emission at $580-620 \mathrm{~nm}$. Quantification was performed using Leica confocal software.

Staining. Suspension cells and root-tip cells of $P$. tomentos $a$ were incubated in $0.3 \mu \mathrm{M}$ CMXRos in slice buffer $\left(0.3 \mathrm{mM}\right.$ sucrose, $5 \mathrm{mM}$ TES, $\left.5 \mathrm{mM} \mathrm{MgCl}_{2}\right)$ at $30^{\circ} \mathrm{C}$ in the dark for $10 \mathrm{~min}$ as described by Lord et al. ${ }^{46}$. Suspension cells and root-tip cells of $P$. tomentosa were incubated in $100 \mu \mathrm{M} \mathrm{JC}-1$ for $20 \mathrm{~min}$ as described by Wang et al. ${ }^{62}$.

Isolation of mitochondria and chloroplast. Mitochondria or chloroplasts were isolated from leaves of P. tomentosa. The purity of the preparations was detected using cytochrome c oxidase for mitochondria, catalase for peroxisomes, alcohol dehydrogenase for cytosol, and alkaline pyrophosphatase for plastids (Supplemental

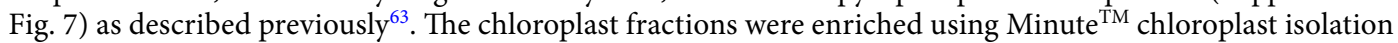
kit(Invent Biotechnologies).

Determination of $\mathrm{H}_{2} \mathrm{O}_{2}$ and MDA contents, ASA/DHA, GSH/GSSG, and ATP/ADP ratios, and ETC complex activity. $\mathrm{H}_{2} \mathrm{O}_{2}$ content was detected using Fluorimetric Hydrogen Peroxide Assay Kit (Sigma-Aldrich). MDA content was detected using Lipid Peroxidation (MDA) Assay Kit (Sigma-Aldrich). ADP and ATP levels were detected using a EnzyLight ${ }^{\mathrm{TM}}$ ATP assay (BioAssay Systems). The levels of AsA, GSSG and GSH were detected as described previously ${ }^{4,64}$.

Immunoblotting. The appearance of carbonyl groups in proteins was detected using an OxiSelect ${ }^{\mathrm{TM}}$ Protein Carbonyl Immunoblot Kit (Cell Biolabs, Inc.).

Flow cytometry. Suspension cells of $P$. tomentosa were incubation for $30 \mathrm{~min}$ in the dark with binding buffer (10 mM HEPES [pH 7.4], $140 \mathrm{mM} \mathrm{NaCl}, 1 \mathrm{mM} \mathrm{MgCl}_{2}, 5 \mathrm{mM} \mathrm{KCl}, 2.5 \mathrm{mM} \mathrm{CaCl} 2,1 \mu \mathrm{g} / \mathrm{mL}$ Hoechst and $1 \mu \mathrm{g} / \mathrm{mL}$ $\mathrm{PI})$ and analyzed on a FACSCalibur flow cytometer (BD Biosciences).

RNA-Seq sample collection, Illumina sequencing, and data processing. Total RNA was isolated from WT, anti-3, and OX cells using TRIzol reagent according to the manufacturer's protocol (Invitrogen). The samples were sequenced using an Illumina Genome Analyzer (HiSeq ${ }^{\text {тм }}$ 2000; Illumina, San Diego, CA). The raw reads were data-filtered to obtain high-quality clean reads. The clean reads were mapped to the P. trichocarpa reference genome and reference genes using SOAPaligner/SOAP2. No more than two mismatches were allowed in the alignment. Gene expression levels were calculated as reads per kilobase per million reads (RPKM) ${ }^{65}$. The data are deposited in the National Center for Biotechnology Information Gene Expression Omnibus database (https:// www.ncbi.nlm.nih.gov/geo/query/acc.cgi?acc=GSE121562) under accession number GSE121562.

Identification and analysis of differentially expressed genes. Differentially expressed genes (DEGs) among WT, anti-3, and OX cells were identified using the DEGseq R package (1.12.0) $)^{65}$ based on the normalized read counts. A corrected P-value $<0.001$ and $\mid \log 2$ ratio $\mid>1.5$ were the thresholds for significantly different expression. GO enrichment analyses of the DEGs were performed using the GOseq R package ${ }^{66}$. GO terms with a P-value $<0.005$ were considered significantly enriched in DEGs.

Accession numbers. Sequence data from this article can be found in the NCBI database under accession numbers MH910690 (PtomtAPX) and MH910610 (PtosAPX), AB022273 (Nt.tAPX), AB114856 (Os.tAPX), D77997 (So.tAPX), D83656 (Cka.tAPX), AB022274 (Nt.sAPX), AB114855 (Os.sAPX), D83669 (So.sAPX) and D88420 (Cka.sAPX).

Received: 11 April 2019; Accepted: 6 December 2019;

Published online: 20 December 2019

\section{References}

1. Considine, M. J. \& Foyer, C. H. Redox regulation of plant development. Antioxid Redox Signal. 21, 1305-1326 (2014).

2. Anjum, N. A. et al. Catalase and ascorbate peroxidase-representative $\mathrm{H}_{2} \mathrm{O}_{2}$-detoxifying heme enzymes in plants. Environ Sci Pollut Res Int. 23, 19002-19029 (2016).

3. Sofo, A., Scopa, A., Nuzzaci, M. \& Vitti, A. Ascorbate peroxidase and catalase activities and their genetic regulation in plants subjected to drought and salinity stresses. Int J Mol Sci. 16, 13561-13578 (2015).

4. Chew, O., Rudhe, C., Glaser, E. \& Whelan, J. Characterization of the targeting signal of dual-targeted pea glutathione reductase. Plant Mol Biol. 53, 341-356 (2003).

5. Teixeira, F. K., Menezes-Benavente, L., Margis, R. \& Margis-Pinheiro, M. Analysis of the molecular evolutionary history of the ascorbate peroxidase gene family: inferences from the rice genome. J Mol Evol. 59, 761-770 (2004).

6. Teixeira, F. K., Menezes-Benavente, L., Galvão, V. C., Margis, R. \& Margis-Pinheiro, M. Rice ascorbate peroxidase gene family encodes functionally diverse isoforms localized in different subcellular compartments. Planta 224, 300-314 (2006).

7. Najami, N. et al. Ascorbate peroxidase gene family in tomato: its identification and characterization. Mol Genet Genomics. 279, 171-182 (2008)

8. Pnueli, L., Liang, H., Rozenberg, M. \& Mittler, R. Growth suppression, altered stomatal responses, and augmented induction of heat shock proteins in cytosolic ascorbate peroxidase (Apx1)-deficient Arabidopsis plants. Plant J 34, 187-203 (2003).

9. Davletova, S. et al. Cytosolic ascorbate peroxidase 1 is a central component of the reactive oxygen gene network of Arabidopsis. Plant Cell 17, 268-281 (2005).

10. Koussevitzky, S. et al. Ascorbate peroxidase 1 plays a key role in the response of Arabidopsis thaliana to stress combination. J Biol Chem 283, 34197-34203 (2008).

11. Narendra, S. et al. The Arabidopsis ascorbate peroxidase 3 is a peroxisomal membrane-bound antioxidant enzyme and is dispensable for Arabidopsis growth and development. J Exp Bot 57, 3033-3042 (2006). 
12. Miller, G. et al. Double mutants deficient in cytosolic and thylakoid ascorbate peroxidase reveal a complex mode of interaction between reactive oxygen species, plant development, and response to abiotic stresses. Plant Physiol 144, 1777-1785 (2007).

13. Chen, C. et al. ASCORBATE PEROXIDASE6 protects Arabidopsis desiccating and germinating seeds from stress and mediates cross talk between reactive oxygen species, abscisic acid, and auxin. Plant Physiol. 166, 370-83 (2014).

14. Rosa, S. B. et al. Cytosolic APx knockdown indicates an ambiguous redox responses in rice. Phytochemistry 71, 548-558 (2010).

15. Bonifacio, A. et al. Role of peroxidases in the compensation of cytosolic ascorbate peroxidase knockdown in rice plants under abiotic stress. Plant Cell Environ 34, 1705-1722 (2011).

16. Zhang, Z. et al. Gene knockout study reveals that cytosolic ascorbate peroxidase 2(OsAPX2) plays a critical role in growth and reproduction in rice under drought, salt and cold stresses. PLoS One. 8, e57472 (2013).

17. Ribeiro, C. W. et al. Rice peroxisomal ascorbate peroxidase knockdown affects ROS signaling and triggers early leaf senescence. Plant Sci. 263, 55-65 (2017).

18. Jimenez, A., Hernandez, J. A., Pastori, G., del Rio, L. A. \& Sevilla, F. Role of the ascorbate-glutathione cycle of mitochondria and peroxisomes in the senescence of pea leaves. Plant Physiol. 118, 1327-1335 (1998).

19. Mittova, V. et al. Comparison of mitochondrial ascorbate peroxidase in the cultivated tomato, Lycopersicon esculentum, and its wild, salt-tolerant relative, $L$. pennellii- a role for matrix isoforms in protection against oxidative damage. Plant Cell Environ. 27, 237-250 (2004).

20. De Leonardis, S., Dipierro, N. \& Dipierro, S. Purification and characterization of an ascorbate peroxidase from potato tuber mitochondria. Plant Physiol Biochem. 38, 773-779 (2000).

21. Khanna-Chopra, R., Jajoo, A. \& Semwal, V. K. Chloroplasts and mitochondria have multiple heat tolerant isozymes of SOD and APX in leaf and inflorescence in Chenopodium album. Biochem Biophys Res Commun. 412, 522-525 (2011).

22. Mittler, R., Vanderauwera, S., Gollery, M. \& Van Breusegem, F. Reactive oxygen gene network of plants. Trends Plant Sci. 9, 490-498 (2004).

23. Giacomelli, L., Masi, A., Ripoll, D. R., Lee, M. J. \& van Wijk, K. J. Arabidopsis thaliana deficient in two chloroplast ascorbate peroxidases shows accelerated light-induced necrosis when levels of cellular ascorbate are low. Plant Mol Biol. 65, 627-644 (2007).

24. Kangasjärvi, S. et al. Diverse roles for chloroplast stromal and thylakoid-bound ascorbate peroxidases in plant stress responses. Biochem J. 412, 275-285 (2008).

25. Caverzan, A. et al. The knockdown of chloroplastic ascorbate peroxidases reveals its regulatory role in the photosynthesis and protection under photo-oxidative stress in rice. Plant Sci. 214, 74-87 (2014).

26. Ge, C., Spånning, E., Glaser, E. \& Wieslander, A. Import determinants of organelle-specific and dual targeting peptides of mitochondria and chloroplasts in Arabidopsis thaliana. Mol Plant. 7, 121-136 (2014).

27. Krause, M. \& Durner, J. Harpin inactivates mitochondria in Arabidopsis suspension cells. Mol Plant Microbe Interact. 17, 131-139 (2004).

28. Hoeberichts, F. A. \& Woltering, E. J. Multiple mediators of plant programmed cell death: interplay of conserved cell death mechanisms and plant-specific regulators. Bioessays 25, 47-57 (2003).

29. Van Aken, O. \& Van Breusegem, F. Licensed to kill: mitochondria, chloroplasts, and cell death. Trends Plant Sci. 20, 754-766 (2015).

30. Cheng, M. C. et al. Increased glutathione contributes to stress tolerance and global translational changes in Arabidopsis. Plant J. 83, 926-939 (2015)

31. Guimier, A. et al. Biallelic PPA2 Mutations Cause Sudden Unexpected Cardiac Arrest in Infancy. Am J Hum Genet. 99, 666-673 (2016).

32. Honda, A. et al. Phosphatidylinositol 4-phosphate 5-kinase alpha is a downstream effector of the small G protein ARF6 in membrane ruffle formation. Cell 99, 521-532 (1999).

33. Mortier, V. et al. Role of LONELY GUY genes in indeterminate nodulation on Medicago truncatula. New Phytol. 202, 582-593 (2014).

34. Woo, H. H., Orbach, M. J., Hirsch, A. M. \& Hawes, M. C. Meristem-localized inducible expression of a UDP-glycosyltransferase gene is essential for growth and development in pea and alfalfa. Plant Cell 11, 2303-2315 (1999).

35. Wang, J., Ma, X. M., Kojima, M., Sakakibara, H. \& Hou, B. K. Glucosyltransferase UGT76C1 finely modulates cytokinin responses via cytokinin N-glucosylation in Arabidopsis thaliana. Plant Physiol Biochem. 65, 9-16 (2013).

36. Lee, R. H., Lin, M. C. \& Chen, S. C. A novel alkaline alpha-galactosidase gene is involved in rice leaf senescence. Plant Mol Biol. 55, 281-295 (2004)

37. Henzler, T. \& Steudle, E. Transport and metabolic degradation of hydrogen peroxide in Chara corallina: model calculations and measurements with the pressure probe suggest transport of $\mathrm{H}_{2} \mathrm{O}_{2}$ across water channels. J Exp Bot. 51, 2053-2066 (2000).

38. Tahara, E. B., Navarete, F. D. \& Kowaltowski, A. J. Tissue-, substrate-, and site-specific characteristics of mitochondrial reactive oxygen species generation. Free Radic Biol Med. 46, 1283-1297 (2009).

39. Melser, S., Lavie, J. \& Bénard, G. Mitochondrial degradation and energy metabolism. Biochim Biophys Acta. 1853, 2812-2821 (2015).

40. Chotewutmontri, P., Reddick, L. E., McWilliams, D. R., Campbell, I. M. \& Bruce, B. D. Differential transit peptide recognition during preprotein binding and translocation into flowering plant plastids. Plant Cell 24, 3040-3059 (2012).

41. Teixeira, P. F. \& Glaser, E. Processing peptidases in mitochondria and chloroplasts. Biochim Biophys Acta. 1833, 360-370 (2013).

42. Bhushan, S., Kuhn, C., Berglund, A. K., Roth, C. \& Glaser, E. The role of the N-terminal domain of chloroplast targeting peptides in organellar protein import and mis-sorting. FEBS Lett. 580, 3966-3972 (2006).

43. Moberg, P. et al. NMR solution structure of the mitochondrial F1beta presequence from Nicotiana plumbaginifolia. J Mol Biol. 336, 1129-1140 (2004).

44. Xu, L., Carrie, C., Law, S. R., Murcha, M. W. \& Whelan, J. Acquisition, conservation, and loss of dual-targeted proteins in land plants. Plant Physiol. 161, 644-662 (2013).

45. Peeters, N. \& Small, I. Dual targeting to mitochondria and chloroplasts. Biochim Biophys Acta. 1541, 54-63 (2001).

46. Carrie, C. \& Small, I. A reevaluation of dual-targeting of proteins to mitochondria and chloroplasts. Biochim Biophys Acta. 1833, 253-259 (2013).

47. Berglund, A. K. et al. Dual targeting to mitochondria and chloroplasts: characterization of Thr-tRNA synthetase targeting peptide. Mol Plant. 2, 1298-1309 (2009).

48. Dinur-Mills, M., Tal, M. \& Pines, O. Dual targeted mitochondrial proteins are characterized by lower MTS parameters and total net charge. PloS One 3, e2161 (2008).

49. Dinakar, C., Abhaypratap, V., Yearla, S. R., Raghavendra, A. S. \& Padmasree, K. Importance of ROS and antioxidant system during the beneficial interactions of mitochondrial metabolism with photosynthetic carbon assimilation. Planta 231, 461-474 (2010).

50. Sun, H., Li, L., Wang, X., Wu, S. \& Wang, X. Ascorbate-glutathione cycle of mitochondria in osmoprimed soybean cotyledons in response to imbibitional chilling injury. J Plant Physiol. 168, 226-232 (2011).

51. Fryer, M. J. et al. Control of Ascorbate Peroxidase 2 expression by hydrogen peroxide and leaf water status during excess light stress reveals a functional organisation of Arabidopsis leaves. Plant J. 33, 691-705 (2003).

52. Yoshimura, K., Yabuta, Y., Ishikawa, T. \& Shigeoka, S. Expression of spinach ascorbate peroxidase isoenzymes in response to oxidative stresses. Plant Physiol. 123, 223-234 (2000).

53. Maruta, T. et al. Arabidopsis chloroplastic ascorbate peroxidase isoenzymes play a dual role in photoprotection and gene regulation under photooxidative stress. Plant Cell Physiol. 51, 190-200 (2010). 
54. Yao, N., Eisfelder, B. J., Marvin, J. \& Greenberg, J. T. The mitochondrion-an organelle commonly involved in programmed cell death in Arabidopsis thaliana. Plant J. 40, 596-610 (2004).

55. Lord, C. E., Wertman, J. N., Lane, S. \& Gunawardena, A. H. Do mitochondria play a role in remodelling lace plant leaves during programmed cell death? BMC Plant Biol. 11, 102 (2011).

56. Okamoto, K., Kondo-Okamoto, N. \& Ohsumi, Y. Mitochondria-anchored receptor Atg32 mediates degradation of mitochondria via selective autophagy. Dev Cell. 17, 87-97 (2009).

57. Ozyigit, I. I. et al. Identification and comparative analysis of $\mathrm{H}_{2} \mathrm{O}_{2}$-scavenging enzymes (ascorbate peroxidase and glutathione peroxidase) in selected plants employing bioinformatics approaches. Front Plant Sci. 22, 301 (2016).

58. Zabka, A., Winnicki, K., Polit, J. T. \& Maszewski, J. Sanguinarine-induced oxidative stress and apoptosis-like programmed cell death(AL-PCD) in root meristem cells of Allium cepa. Plant Physiol Biochem. 112, 193-206 (2017).

59. Li, Y. et al. Stable transformation of suspension-cultured Glycyrrhiza inflata batalin cells with Agrobacterium tumefaciens. $Z$ Naturforsch C. 67, 603-610 (2012).

60. Zhang, X. M. et al. NtCP56, a new cysteine protease in Nicotiana tabacum L., involved in pollen grain development. J Exp Bot. 60, 1569-1577 (2009).

61. Zhang, D. et al. The cysteine protease CEP1, a key executor involved in tapetal programmed cell death, regulates pollen development in Arabidopsis. Plant Cell 26, 2939-2961 (2014).

62. Wang, Y., Li, Y., Xue, H., Pritchard, H. W. \& Wang, X. Reactive oxygen species-provoked mitochondria-dependent cell death during ageing of elm (Ulmus pumila L.) seeds. Plant J. 81, 438-452 (2015).

63. Millar, A. H., Sweetlove, L. J., Giegé, P. \& Leaver, C. J. Analysis of the Arabidopsis mitochondrial proteome. Plant Physiol. 127, 1711-1727 (2001).

64. Sultana, A. et al. Catalases CAT1 and CAT3 are not key enzymes in alleviating gamma irradiation-induced DNA damage, $\mathrm{H}_{2} \mathrm{O}_{2}$ accumulation, or lipid peroxidation in Arabidopsis thaliana. Biosci Biotechnol Biochem. 77, 1984-1987 (2013).

65. Wang, Z., Gerstein, M. \& Snyder, M. RNA-Seq: a revolutionary tool for transcriptomics. Nat Rev Genet. 10, 57-63 (2009).

66. Young, M. D., Wakefield, M. J., Smyth, G. K. \& Oshlack, A. Gene ontology analysis for RNA-seq: accounting for selection bias. Genome Biol. 11, R14 (2010).

\section{Acknowledgements}

This work was supported by the Fundamental Research Funds for the Central Universities (JC2015-01) and a grant from the Natural Science Foundation of China (31570582).

\section{Author contributions}

Di Liu and Hai Lu designed the experiments; Bin Yin, Jiaxue Zhang, Yadi Liu and Xiang Pan performed the experiments; Zhijing Zhao, Hui Li, Chong Zhang, Conghui Li, Xihua Du and Yinjun Li performed statistical analysis and prepared tables and figures. Bin Yin, Di Liu and Hai Lu wrote the manuscript.

\section{Competing interests}

The authors declare no competing interests.

\section{Additional information}

Supplementary information is available for this paper at https://doi.org/10.1038/s41598-019-56148-w.

Correspondence and requests for materials should be addressed to D.L. or H.L.

Reprints and permissions information is available at www.nature.com/reprints.

Publisher's note Springer Nature remains neutral with regard to jurisdictional claims in published maps and institutional affiliations.

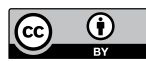

Open Access This article is licensed under a Creative Commons Attribution 4.0 International

License, which permits use, sharing, adaptation, distribution and reproduction in any medium or format, as long as you give appropriate credit to the original author(s) and the source, provide a link to the Creative Commons license, and indicate if changes were made. The images or other third party material in this article are included in the article's Creative Commons license, unless indicated otherwise in a credit line to the material. If material is not included in the article's Creative Commons license and your intended use is not permitted by statutory regulation or exceeds the permitted use, you will need to obtain permission directly from the copyright holder. To view a copy of this license, visit http://creativecommons.org/licenses/by/4.0/.

(C) The Author(s) 2019 